\title{
MONADIC INTUITIONISTIC AND MODAL LOGICS ADMITTING PROVABILITY INTERPRETATIONS
}

\author{
GURAM BEZHANISHVILI, KRISTINA BRANTLEY, AND JULIA ILIN
}

\begin{abstract}
The Gödel translation provides an embedding of the intuitionistic logic IPC into the modal logic Grz, which then embeds into the modal logic GL via the splitting translation. Combined with Solovay's theorem that GL is the modal logic of the provability predicate of Peano Arithmetic PA, both IPC and Grz admit arithmetical interpretations. When attempting to 'lift' these results to the monadic extensions MIPC, MGrz, and MGL of these logics, the same techniques no longer work. Following a conjecture made by Esakia, we add an appropriate version of Casari's formula to these monadic extensions (denoted by a ' +'), obtaining that the Gödel translation embeds $M^{+} I P C$ into $M^{+} G r z$ and the splitting translation embeds $M^{+}$Grz into MGL. As proven by Japaridze, Solovay's result extends to the monadic system MGL, which leads us to an arithmetical interpretation of both $\mathrm{M}^{+} \mathrm{IPC}$ and $\mathrm{M}^{+} \mathrm{Grz}$.
\end{abstract}

\section{CONTENTS}

1. Introduction

1.1. Propositional case

1.2. Predicate case

1.3. Monadic case

1.4. Main contribution and organization

2. Monadic logics

2.1. Monadic intuitionistic logic

2.2. Monadic modal logics

2.3. MS4, MGrz, and MGL

3. The Gödel and splitting translations in the monadic setting

3.1. Gödel translation

3.2. Splitting translation

4. The logics $\mathrm{M}^{+} \mathrm{IPC}$ and $\mathrm{M}^{+} \mathrm{Grz}$

4.1. $\mathrm{M}^{+} \mathrm{IPC}$

4.2. $\mathrm{M}^{+} \mathrm{Grz}$

4.3. The translations $\mathrm{M}^{+} \mathrm{IPC} \rightarrow \mathrm{M}^{+} \mathrm{Grz} \rightarrow \mathrm{MGL}$

5. The finite model property of $\mathrm{M}^{+} \mathrm{IPC}$

5.1. The construction

5.2. Auxiliary lemmas [18

5.3. Termination of the construction $\quad 20$

5.4. Truth lemma 22

6. The finite model property of $\mathrm{M}^{+} \mathrm{Grz}$

6.1. Step 1: Constructing $\mathfrak{F}_{1}$

6.2. Step 2: Constructing $\mathfrak{F}_{2}$

6.3. Step 3: Constructing $\mathfrak{F}_{3}$

References 30

\section{INTRODUCTION}

1.1. Propositional case. It is well known that the Gödel translation embeds Intuitionistic Propositional Calculus IPC into the modal logic S4. We recall that the Gödel translation is defined as follows: 
- $p^{t}=\square p$ for a propositional letter $p$;

- $(\varphi \vee \psi)^{t}=\varphi^{t} \vee \psi^{t}$

- $(\varphi \wedge \psi)^{t}=\varphi^{t} \wedge \psi^{t}$

- $(\varphi \rightarrow \psi)^{t}=\square\left(\varphi^{t} \rightarrow \psi^{t}\right)$;

- $(\neg \varphi)^{t}=\square\left(\neg \varphi^{t}\right)$.

McKinsey and Tarski [26] proved that this translation is full and faithful; that is,

$$
\text { IPC } \vdash \varphi \text { iff } \mathrm{S} 4 \vdash \varphi^{t} \text {. }
$$

There are many other normal extensions of S4, called modal companions of IPC, in which IPC is embedded fully and faithfully. Esakia [12] showed that the largest such companion is Grzegorczyk's logic Grz, which is the normal extension of S4 with the Grzegorczyk axiom

$$
\operatorname{grz}=: \square(\square(p \rightarrow \square p) \rightarrow p) \rightarrow p .
$$

Thus, we have

$$
\text { IPC } \vdash \varphi \text { iff Grz } \vdash \varphi^{t} \text {. }
$$

Goldblatt [21], Boolos [6], and Kuznetsov and Muravitsky [25] showed that the splitting translation embeds Grz into the Gödel-Löb logic GL which is the normal extension of the least normal modal logic K with the axiom

$$
\mathrm{gl}:=\square(\square p \rightarrow p) \rightarrow \square p .
$$

We recall that the splitting translation is defined by "splitting boxes" in formulas (see, e.g., [7, p. 8]); that is, for a modal formula $\varphi$, let $\square^{+} \varphi$ be the abbreviation of the formula $\varphi \wedge \square \varphi$. Then the splitting translation is defined by letting $\varphi^{s}$ be the result of replacing all occurrences of $\square$ in $\varphi$ by $\square^{+}$. We then have

$$
\mathrm{Grz} \vdash \varphi \text { iff GL} \vdash \varphi^{s} .
$$

Combining these results yields

$$
\mathrm{IPC} \vdash \varphi \text { iff Grz } \vdash \varphi^{t} \text { iff GL } \vdash\left(\varphi^{t}\right)^{s} .
$$

By Solovay's theorem 34, GL can be thought of as the modal logic of the provability predicate in Peano Arithmetic PA. Thus, both IPC and Grz admit provability interpretations.

1.2. Predicate case. The Gödel translation extends to the predicate case by setting

- $(\forall x \varphi)^{t}=\square \forall x\left(\varphi^{t}\right)$;

- $(\exists x \varphi)^{t}=\exists x\left(\varphi^{t}\right)$.

Let IQC be the intuitionistic predicate calculus and QS4 the predicate S4. Then

$$
\text { IQC } \vdash \varphi \text { iff QS4 } \vdash \varphi^{t},
$$

so the extension of the Gödel translation to the predicate case remains full and faithful (see, e.g., [33]). However, this is virtually the only positive result. Let QGrz be the predicate Grz and let QGL be the predicate GL. Montagna [27] showed that Solovay's theorem no longer holds for QGL. Moreover, the splitting translation does not embed QGrz fully and faithfully into QGL (see below), and as far as we know, it remains an open problem whether the Gödel translation embeds IQC fully and faithfully into QGrz.

1.3. Monadic case. In view of the above, Esakia [15] suggested to study these translations for the monadic (one-variable) fragments of IQC, QGrz, and QGL. The monadic fragment of IQC was introduced by Prior 32 ] under the name of MIPC. The monadic fragment of QS4 was studied by Fischer-Servi [17, and the monadic fragments of QGrz and QGL by Esakia [15. We denote them by MS4, MGrz, and MGL, respectively.

Fischer-Servi [17] proved that the Gödel translation embeds MIPC into MS4 fully and faithfully. As we will see, the Gödel translation also embeds MIPC fully and faithfully into MGrz. Japaridze 23, 24, proved that Solovay's result extends to MGL. Therefore, to complete the picture, it would be sufficient to show that the splitting translation embeds MGrz into MGL fully and faithfully. However, as was observed by Esakia, this is no longer true. To see this, we recall (see, e.g., [30]) that Casari's formula

$$
\text { Cas : } \forall x((P(x) \rightarrow \forall y P(y)) \rightarrow \forall y P(y)) \rightarrow \forall x P(x)
$$


is valid in an intuitionistic predicate Kripke frame provided the underlying poset is Noetherian. Consider the monadic version of Casari's formula

$$
\text { MCas : } \forall((p \rightarrow \forall p) \rightarrow \forall p) \rightarrow \forall p \text {. }
$$

Using the same notation for the Gödel and splitting translations in the monadic setting, we have that MGrz $\nvdash$ (MCas) $^{t}$ but MGL $\vdash\left((\text { MCas })^{t}\right)^{s}$. This yields that MGrz does not embed into MGL faithfully.

Let

$$
\mathrm{M}^{+} \mathrm{IPC}=\mathrm{MIPC}+\mathrm{MCas}
$$

be the extension of MIPC by MCas, and let

$$
\mathrm{M}^{+} \mathrm{Grz}=\mathrm{MGrz}+(\mathrm{MCas})^{t}
$$

be the extension of MGrz by (MCas) ${ }^{t}$. Esakia claimed that the translations

$$
\mathrm{IPC} \rightarrow \mathrm{Grz} \rightarrow \mathrm{GL}
$$

are lifted to

$$
\mathrm{M}^{+} \mathrm{IPC} \rightarrow \mathrm{M}^{+} \mathrm{Grz} \rightarrow \mathrm{MGL} .
$$

Verifying this claim will be our main goal.

1.4. Main contribution and organization. Our main result is the following theorem.

Theorem. $\mathrm{M}^{+} \mathrm{IPC} \vdash \varphi$ iff $\mathrm{M}^{+} \mathrm{Grz} \vdash \varphi^{t}$ iff $\mathrm{MGL} \vdash\left(\varphi^{t}\right)^{s}$.

We will prove the theorem semantically. The most challenging part of our argument is in establishing the finite model property for $\mathrm{M}^{+} \mathrm{IPC}$ and $\mathrm{M}^{+} \mathrm{Grz}$ (see Sections 5 and 6). It was established by Japaridze [23] that MGL also has the finite model property. In fact, our technique of proving the finite model property for $\mathrm{M}^{+} \mathrm{Grz}$ can be adapted to provide an alternative proof of Japaridze's result for MGL, but this is not needed for the above theorem.

The paper is organized as follows. Section 2 provides a brief overview of monadic logics and their corresponding algebraic and relational semantics. Section 3 discusses the Gödel and splitting translations in the monadic setting. In Section 4 we investigate how the addition of the adapted variations of Casari's formula affect the semantics. In Sections 5 and 6 we establish the finite model property for $\mathrm{M}^{+} \mathrm{IPC}$ and $\mathrm{M}^{+} \mathrm{Grz}$, respectively, using a modified selective filtration, which allows us to conclude the main result stated above.

We use [10] as our standard reference for intuitionistic and modal propositional logic, and [20] as our standard reference for intuitionistic modal logics and classical bi-modal systems.

\section{MONADIC LOGICS}

In this section we recall the notion of monadic intuitionistic and modal logics and discuss their algebraic and frame-based semantics.

2.1. Monadic intuitionistic logic. The monadic intuitionistic propositional calculus MIPC was defined by Prior [32] and it was shown by Bull [9] that MIPC axiomatizes the monadic fragment of the predicate intuitionistic logic. To define MIPC, let $\mathcal{L}$ be the language of propositional intuitionistic logic, and let $\mathcal{L}_{\forall \exists}$ be the enrichment of $\mathcal{L}$ with the quantifier modalities $\forall$ and $\exists]^{1}$

Definition 2.1. MIPC is the smallest set of $\mathcal{L}_{\forall \exists}$-formulas containing

- all axioms of IPC,

- the S4-axioms for $\forall ?^{2}$

- the S5-axioms for $\exists 3^{3}$

- the connecting axioms $\exists p \rightarrow \forall \exists p$ and $\exists \forall p \rightarrow \forall p$,

and closed under the inference rules of substitution, modus ponens, and $\forall$-necessitation $\frac{\varphi}{\forall \varphi}$.

Remark 2.2. The non-symmetric feature of intuitionistic quantifiers is captured in the fact that while $\exists$ is an S5-modality, $\forall$ is merely an S4-modality, and the $\forall$-counterpart $\forall(\forall p \vee q) \leftrightarrow(\forall p \vee \forall q)$ of $\exists(\exists p \wedge q) \leftrightarrow(\exists p \wedge \exists q)$ is not provable in MIPC.

\footnotetext{
${ }^{1} \square$ and $\diamond$ are also frequently used in place of $\forall$ and $\exists$, respectively.

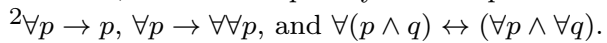

${ }^{3} p \rightarrow \exists p, \exists \exists p \rightarrow \exists p, \exists(p \vee q) \leftrightarrow(\exists p \vee \exists q)$, and $\exists(\exists p \wedge q) \leftrightarrow(\exists p \wedge \exists q)$.
} 
Algebraic semantics for MIPC is given by monadic Heyting algebras [28, 2].

Definition 2.3. A monadic Heyting algebra is a triple $(H, \forall, \exists)$ where

- $H$ is a Heyting algebra,

- $\forall: H \rightarrow H$ is an S4-operator ${ }^{4}$

- $\exists: H \rightarrow H$ is an S5-operator 5

- $\exists a \leq \forall \exists a$ and $\exists \forall a \leq \forall a$.

Remark 2.4. This in particular implies that the fixpoints of $\forall$ and $\exists$ are equal and form a Heyting subalgebra of $H$. In fact, every monadic Heyting algebra can be represented as a pair $\left(H, H_{0}\right)$ where $H_{0}$ is a Heyting subalgebra of $H$ and the inclusion has both the right $(\forall)$ and left $(\exists)$ adjoint.

As usual, propositional letters of $\mathcal{L}_{\forall \exists}$ are evaluated as elements of $H$, the connectives as the corresponding operations of $H$, and the quantifier modalities as the corresponding modal operators of $H$. The standard Lindenbaum-Tarski construction then yields:

Theorem 2.5. MIPC $\vdash \varphi \Leftrightarrow \mathfrak{H} \vDash \varphi$ for each monadic Heyting algebra $\mathfrak{H}$.

Kripke semantics for MIPC is an extension of Kripke semantics for IPC [29, 19, 15].

Definition 2.6. An MIPC-frame is a triple $\mathfrak{F}=(W, R, E)$ where $(W, R)$ is an IPC-frame ${ }^{6}$ and $E$ is an equivalence relation on $W$ satisfying $(R \circ E)(x) \subseteq(E \circ R)(x)$ for all $x \in W$; that is, if $x E y$ and $y R z$, then there is $w \in W$ such that $x R w$ and $w E z$.

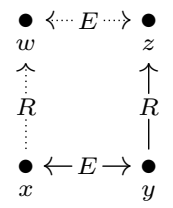

We refer to this condition as commutativity. We will sometimes refer to $R$ as a 'vertical relation', and to $E$ as a 'horizontal relation', as depicted in the diagram above.

Valuations on MIPC-frames are defined as for IPC-frames; that is, a valuation on $\mathfrak{F}=(W, R, E)$ is an assignment $\nu$ of propositional letters to $R$-upsets of $\mathfrak{F} 7$ As usual, the truth relation in $\mathfrak{F}$ is defined by induction. The clauses for the connectives $\wedge, \vee, \rightarrow, \neg$ are the same as for IPC-frames:

$$
\begin{array}{lll}
w \vDash_{\nu} p & \text { iff } & w \in \nu(p) ; \\
w \vDash_{\nu} \varphi \wedge \psi & \text { iff } & w \vDash_{\nu} \varphi \text { and } w \vDash_{\nu} \psi ; \\
w \vDash_{\nu} \varphi \vee \psi & \text { iff } & w \vDash_{\nu} \varphi \text { or } w \vDash_{\nu} \psi ; \\
w \vDash_{\nu} \varphi \rightarrow \psi & \text { iff } & (\text { for all } v)\left(w R v \text { and } v \vDash_{\nu} \varphi \text { implies } v \vDash_{\nu} \psi\right. \text { ); } \\
w \vDash_{\nu} \neg \varphi & \text { iff } & \text { (for all } v)\left(w R v \text { implies } v \not F_{\nu} \varphi\right) .
\end{array}
$$

To extend this to the truth relation for quantifier modalities, we first define a new relation $Q$ as the composition $R \circ E$ on $W$; that is, $x Q y$ iff there is $z \in W$ such that $x R z$ and $z E y$.

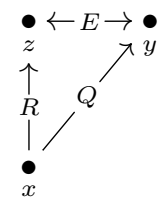

Then $Q$ is a quasi-order (reflexive and transitive) and $\forall, \exists$ are interpreted in $\mathfrak{F}$ as follows:

$$
\begin{array}{lll}
w \vDash_{\nu} \forall \varphi & \text { iff } & \text { (for all } v)\left(w Q v \text { implies } v \vDash_{\nu} \varphi\right) ; \\
w \vDash_{\nu} \exists \varphi & \text { iff } & \text { (there exists } v)\left(w E v \text { and } v \vDash_{\nu} \varphi\right) .
\end{array}
$$

${ }^{4} \forall a \leq a, \forall a \leq \forall \forall a, \forall(a \wedge b)=\forall a \wedge \forall b$, and $\forall 1=1$.

${ }^{5} a \leq \exists a, \exists \exists a \leq \exists a, \exists(a \vee b)=\exists a \vee \exists b, \exists 0=0$, and $\exists(\exists a \wedge b)=\exists a \wedge \exists b$.

${ }^{6} \mathrm{~A}$ nonempty partially ordered set.

${ }^{7}$ Recall that $U \subseteq W$ is an $R$-upset if $u \in U$ and $u R v$ imply $v \in U$. 
Sometimes we also write $(\mathfrak{F}, w) \vDash_{\nu} \varphi$ to emphasize the underlying frame $\mathfrak{F}$ or simply $w \vDash \varphi$ in case $\mathfrak{F}$ and $\nu$ are clear from the context.

There is a close connection between algebraic and relational semantics for MIPC. To see this, let $\mathfrak{F}=$ $(W, R, E)$ be an MIPC-frame. For $x \in W$, let

$$
Q(x)=\{y \in W \mid x Q y\} \text { and } E(x)=\{y \in W \mid x E y\} .
$$

Set $\mathfrak{F}^{+}=(\mathrm{Up}(\mathfrak{F}), \forall, \exists)$ where $U \mathrm{p}(\mathfrak{F})$ is the Heyting algebra of $R$-upsets of $\mathfrak{F}$, and for $U \in \mathrm{Up}(\mathfrak{F})$,

$$
\forall U=\{x \in W \mid Q(x) \subseteq U\} \text { and } \exists U=\{x \in W \mid E(x) \cap U \neq \varnothing\} .
$$

Then $\mathfrak{F}^{+}$is a monadic Heyting algebra, and every monadic Heyting algebra is represented as a subalgebra of such. To see this, for a monadic Heyting algebra $\mathfrak{H}=(H, \forall, \exists)$, let $W$ be the set of prime filters of $H$, let $R$ be the inclusion, and let $E$ be defined by $\eta E \zeta$ iff $\eta \cap H_{0}=\zeta \cap H_{0}$, where we recall that $H_{0}$ is the fixpoint subalgebra of $H$ (see Remark 2.4). Then $\mathfrak{H}_{+}=\left(W, R, E\right.$ ) is an MIPC-frame (where $\eta Q \zeta$ iff $\eta \cap H_{0} \subseteq \zeta \cap H_{0}$ ) and there is an embedding $e: \mathfrak{H} \rightarrow\left(\mathfrak{H}_{+}\right)^{+}$given by

$$
e(a)=\left\{\eta \in \mathfrak{H}_{+} \mid a \in \eta\right\} .
$$

In general, the embedding $e$ is not onto, so to recognize the $e$-image of $H$ in the Heyting algebra of upsets, we introduce the concept of a descriptive MIPC-frame. One way to do this is to introduce topology on an MIPC-frame.

We recall that a topological space is a Stone space if it is compact Hausdorff and zero-dimensiona 8 . A relation $R$ on a Stone space $W$ is continuous if (i) $R(x)$ is closed for each $x \in W$ and (ii) $U$ clopen implies $R^{-1}(U)$ is clopen, where

$$
R^{-1}(U)=\{x \in W \mid x R u \text { for some } u \in U\} .
$$

Definition 2.7. An MIPC-frame $\mathfrak{F}=(W, R, E)$ is a descriptive MIPC-frame if

- $W$ is a Stone space,

- $R$ and $Q$ are continuous relations,

- $A$ clopen $R$-upset implies $E(A)$ is a clopen $R$-upset.

Remark 2.8. This does not imply that $A$ clopen implies $E(A)$ is clopen; see [3, p. 32]. However, we do have that $A$ closed implies $E(A)$ is closed; see [3, Lem. 7].

As follows from Esakia's representation of Heyting algebras [11, for a Heyting algebra $H$, there is a Stone topology on the set $W$ of prime filters of $H$ generated by the basis

$$
\{e(a) \backslash e(b) \mid a, b \in H\},
$$

the inclusion relation $R$ on $W$ is continuous, and $e$ is a Heyting isomorphism from $H$ onto the Heyting algebra of clopen $R$-upsets of $W$.

By [3, Thm. 13], if $\mathfrak{H}=(H, \forall, \exists)$ is a monadic Heyting algebra, then $(W, R, E)$ is a descriptive MIPC-frame, which we denote by $\mathfrak{H}_{*}$, and $e$ is an isomorphism from $\mathfrak{H}$ onto the monadic Heyting algebra $\left(\mathfrak{H}_{*}\right)^{*}$ of clopen $R$-upsets of $\mathfrak{H}_{*}$. Thus, every monadic Heyting algebra can be thought of as the algebra of clopen $R$-upsets of some descriptive MIPC-frame. This representation together with Theorem 2.5 yields:

Theorem 2.9. MIPC $\vdash \varphi \Leftrightarrow \mathfrak{F} \vDash \varphi$ for each descriptive MIPC-frame $\mathfrak{F}$.

If the descriptive MIPC-frame is finite, then the topology is discrete, and hence finite descriptive MIPCframes are simply finite MIPC-frames. It is well known that MIPC has the finite model property:

Theorem 2.10. MIPC $\vdash \varphi \Leftrightarrow \mathfrak{F} \vDash \varphi$ for each finite MIPC-frame $\mathfrak{F}$.

This was first proved by Bull [8] using algebraic semantics. However, Bull's proof contained a gap, which was later filled by Fischer-Servi [18] and Ono [29] independently of each other. For a more frame-theoretic proof, using the technique of selective filtration, see [20, §10.3].

We finish $\S 2.1$ by recalling an important property of descriptive MIPC-frames, which will be useful later on.

Definition 2.11. Let $\mathfrak{F}=(W, R, E)$ be a descriptive MIPC-frame and let $A \subseteq W$.

(1) We say $x \in A$ is $R$-maximal in $A$ if $x R y$ and $y \in A$ imply $x=y$.

${ }^{8}$ Clopen (closed and open) sets form a basis for the topology. 
(2) The $R$-maximum of $A$ is the set of all $R$-maximal points of $A$, i.e.,

$$
\max A=\{x \in A \mid x R y \text { and } y \in A \text { imply } x=y\} .
$$

The next lemma states that every point in the $E$-saturation of clopen $A$ sees a point that is maximal in the $E$-saturation of $A$. The proof follows from the result of Fine [16] and Esakia [14 that can be phrased as follows: If $A$ is a closed subset of a descriptive IPC-frame, then for each $x \in A$ there is $y \in \max A$ such that $x R y$. Since $A$ clopen implies that $E(A)$ is closed (see Remark 2.8), the proof is a consequence of the Fine-Esakia lemma.

Lemma 2.12. Let $\mathfrak{F}=(W, R, E)$ be a descriptive MIPC-frame. For each clopen $A$ and $x \in E(A)$, there is $y \in \max E(A)$ such that $x R y$.

2.2. Monadic modal logics. Let $\mathcal{M L}$ be the basic propositional modal language (with one modality $\square$ ). As usual, the least normal modal logic will be denoted by $\mathrm{K}$, and normal modal logics are normal extensions of $\mathrm{K}$.

Let $\mathcal{M} \mathcal{L}_{\forall}$ be the bimodal language which enriches $\mathcal{M L}$ with the modality $\forall$. We use the abbreviation $\exists \varphi$ for $\neg \forall \neg \varphi$.

\section{Definition 2.13.}

(1) The monadic $\mathrm{K}$ is the least set of $\mathcal{M} \mathcal{L}_{\forall}$-formulas containing

- the K-axiom for $\square 9^{9}$

- the S5-axioms for $\forall 10$

- the bridge axiom $\square \forall p \rightarrow \forall \square p$,

and closed under $\forall$-necessitation $\frac{\varphi}{\forall \varphi}$ as well as under the usual rules of substitution, modus ponens, and $\square$-necessitation. We denote the monadic $\mathrm{K}$ by MK.

(2) A normal extension of MK is an extension of MK which is closed under both $\square$ - and $\forall$-necessitation. We call normal extensions of MK normal monadic modal logics or simply mm-logics.

(3) Let $\mathrm{L}$ be a normal modal logic (in $\mathcal{M L}$ ). The least monadic extension $\mathrm{ML}$ of $\mathrm{L}$ is the smallest mm-logic containing MK $\cup \mathrm{L}$.

\section{Remark 2.14.}

(1) Monadic modal logics are bimodal logics in the language with two modalities $\square, \forall$, where $\forall$ is an S5-modality. They correspond to expanding relativized products discussed in [20, §9].

(2) The axiom $\forall \square p \rightarrow \square \forall p$, which is the converse of the bridge axiom, and is the monadic version of Barcan's formula, is not provable in MK.

Algebraic semantics for monadic modal logics is given by monadic modal algebras.

Definition 2.15. A monadic modal algebra or simply an mm-algebra is a triple $(B, \square, \forall)$ where

- $(B, \square)$ is a modal algebra 11

- $(B, \forall)$ is an S5-algebra ${ }^{12}$

- $\square \forall a \leq \forall \square a$.

Remark 2.16. As with monadic Heyting algebras, the $\forall$-fixpoints of an mm-algebra $(B, \square, \forall)$ form a subalgebra of the modal algebra $(B, \square)$, and each mm-algebra is represented as a pair $\left(B, B_{0}\right)$ of modal algebras such that the embedding of $B_{0}$ into $B$ has a right adjoint $(\forall)$.

Kripke semantics for mm-logics is given by augmented Kripke frames of Esakia [15].

Definition 2.17. An augmented Kripke frame is a triple $\mathfrak{F}=(W, R, E)$ where $(W, R)$ is a Kripke frame ${ }^{13}$ and $E$ is an equivalence relation on $W$ satisfying commutativity, i.e., $(R \circ E)(x) \subseteq(E \circ R)(x)$ for all $x \in W$;

\footnotetext{
${ }^{9} \square(p \rightarrow q) \rightarrow(\square p \rightarrow \square q)$.

${ }^{10} \forall p \rightarrow p, \forall p \rightarrow \forall \forall p, \neg \forall p \rightarrow \forall \neg \forall p$, and $\forall(p \rightarrow q) \rightarrow(\forall p \rightarrow \forall q)$.

${ }^{11}$ That is, $B$ is a boolean algebra and $\square: B \rightarrow B$ satisfies $\square 1=1$ and $\square(a \wedge b)=\square a \wedge \square b$.

${ }^{12}$ That is, $(B, \forall)$ is a modal algebra satisfying $\forall a \leq a, \forall a \leq \forall \forall a$, and $\neg \forall a \leq \forall \neg \forall a$.

${ }^{13} W$ is nonempty and $R$ is a binary relation on $W$.
} 
that is, if $x E y$ and $y R z$, then there is $w \in W$ such that $x R w$ and $w E z$.

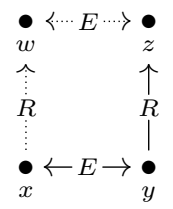

As with MIPC-frames, we may refer to $R$ as a 'vertical relation,' and to $E$ as a 'horizontal relation,' as depicted in the diagram above.

Valuations on augmented Kripke frames are defined analogously to Kripke frames; that is, a valuation $\nu$ on an augmented Kripke frame $\mathfrak{F}=(W, R, E)$ assigns propositional letters to subsets of $W$. The truth relation clauses for the connectives $\vee, \neg$, the modality $\square$, and its dual $\diamond$ are defined as for Kripke frames:

$$
\begin{array}{lll}
x \vDash_{\nu} p & \text { iff } & x \in \nu(p) ; \\
x \vDash_{\nu} \psi \vee \chi & \text { iff } & x \vDash_{\nu} \psi \text { or } x \vDash_{\nu} \chi ; \\
x \vDash_{\nu} \neg \psi & \text { iff } & x \nvdash_{\nu} \psi ; \\
x \vDash_{\nu} \square \psi & \text { iff } & (\text { for all } y \in W)\left(x R y \Rightarrow y \vDash_{\nu} \psi\right) ; \\
x \vDash_{\nu} \diamond \psi & \text { iff } & (\text { there exists } y \in W)\left(x R y \text { and } y \vDash_{\nu} \psi\right) .
\end{array}
$$

The modality $\forall$ and its dual $\exists$ are interpreted via the relation $E$ as follows:

$$
\begin{array}{lll}
x \vDash_{\nu} \forall \varphi & \text { iff } & \text { (for all } y \in W)\left(x E y \Rightarrow y \vDash_{\nu} \varphi\right) \\
x \vDash_{\nu} \exists \varphi & \text { iff } & \text { (there exists } y \in W)\left(x E y \text { and } y \vDash_{\nu} \varphi\right) .
\end{array}
$$

As in the case of MIPC-frames, we also use the notation $(\mathfrak{F}, w) \vDash_{\nu} \varphi$ or $w \vDash \varphi$.

As in the case of MIPC, there is a close connection between algebraic and relational semantics for mmlogics. For an augmented Kripke frame $\mathfrak{F}=(W, R, E)$, set $\mathfrak{F}^{+}=(\wp(\mathfrak{F}), \square, \forall)$ where $\wp(\mathfrak{F})$ is the powerset of $\mathfrak{F}$, and for $U \in U \mathrm{p}(\mathfrak{F})$,

$$
\square U=\{x \in W \mid R(x) \subseteq U\} \text { and } \forall U=\{x \in W \mid E(x) \subseteq U\} .
$$

Then $\mathfrak{F}^{+}$is an mm-algebra, and every mm-algebra is represented as a subalgebra of such. To see this, for an mm-algebra $\mathfrak{B}=(B, \square, \forall)$, let $W$ be the set of ultrafilters of $B$, and let $R$ and $E$ be defined by

$$
\eta R \zeta \text { iff } \square a \in \eta \text { implies } a \in \zeta \text { and } \eta E \zeta \text { iff } \eta \cap B_{0}=\zeta \cap B_{0} .
$$

Then $\mathfrak{B}_{+}=(W, R, E)$ is an augmented Kripke frame and there is an embedding $e: \mathfrak{B} \rightarrow\left(\mathfrak{B}_{+}\right)^{+}$given by

$$
e(a)=\left\{\eta \in \mathfrak{B}_{+} \mid a \in \eta\right\} .
$$

In general, the embedding $e$ is not onto, so to recognize the $e$-image of $\mathfrak{B}$ in the powerset, we introduce the concept of a descriptive augmented Kripke frame. As in the case of MIPC, we do this by introducing topology on augmented Kripke frames.

Definition 2.18. An augmented Kripke frame $\mathfrak{F}=(W, R, E)$ is a descriptive augmented Kripke frame if $W$ is a Stone space and $R$ and $E$ are continuous relations.

As follows from the representation of modal algebras, for a modal algebra $B$, there is a Stone topology on the set $W$ of ultrafilters of $B$ generated by the basis $\{e(a) \mid a \in B\}$, the relation $R$ on $W$ is continuous, and $e$ is a modal isomorphism from $B$ onto the modal algebra of clopen subsets of $W$.

If $\mathfrak{B}=(B, \square, \forall)$ is an mm-algebra, then $(W, R, E)$ is a descriptive augmented Kripke frame, which we denote by $\mathfrak{B}_{*}$, and $e$ is an isomorphism from $\mathfrak{B}$ onto the mm-algebra $\left(\mathfrak{B}_{*}\right)^{*}$ of clopen subsets of $\mathfrak{B}_{*}$. Thus, every mm-algebra can be thought of as the algebra of clopen subsets of some descriptive augmented Kripke frame. 
2.3. MS4, MGrz, and MGL. We next focus on the least monadic extension MS4 of the modal logic S4.

\section{Definition 2.19.}

(1) An MS4-algebra is an mm-algebra $(B, \square, \forall)$ such that $(B, \square)$ is an S4-algebra.

(2) An MS4-frame is an augmented Kripke frame $\mathfrak{F}=(W, R, E)$ such that $(W, R)$ is an S4-frame.

(3) A descriptive MS4-frame is a descriptive augmented Kripke frame $\mathfrak{F}=(W, R, E)$ such that $(W, R, E)$ is an MS4-frame.

As in the case of MIPC, we have the following standard completeness results:

Theorem 2.20.

(1) MS4 $\vdash \varphi \Leftrightarrow \mathfrak{B} \vDash \varphi$ for each MS4-algebra $\mathfrak{B}$.

(2) MS4 $\vdash \varphi \Leftrightarrow \mathfrak{F} \vDash \varphi$ for each descriptive MS4-frame $\mathfrak{F}$.

We also have that MS4 has the finite model property. This can be proved by adopting the algebraic proof of the finite model property of MIPC to the setting of MS4 (see [5]).

Theorem 2.21. MS4 $\vdash \varphi \Leftrightarrow \mathfrak{F} \vDash \varphi$ for each finite MS4-frame $\mathfrak{F}$.

Let $\mathfrak{F}=(W, R, E)$ be a descriptive MS4-frame and $A \subseteq W$. The $R$-maximal points of $A$ and the $R$ maximum of $A$ are defined as in Definition 2.11. In the context of MS4-frames, we also need the notion of quasi- $R$-maximal points.

Definition 2.22. Let $\mathfrak{F}=(W, R, E)$ be a descriptive MS4-frame and $A \subseteq W$.

(1) We say $x \in A$ is quasi-R-maximal in $A$ if $x R y$ and $y \in A$ imply $y R x$.

(2) The quasi-R-maximum of $A$ is the set of all quasi-R-maximal points of $A$, i.e.,

$$
\text { qmax } A=\{x \in A \mid x R y \text { and } y \in A \text { imply } y R x\} \text {. }
$$

Note that $\max A \subseteq$ qmax $A$ as $R$ is reflexive, but not conversely. The following lemma is a consequence of the Fine-Esakia lemma [16, 14, for descriptive S4-frames.

Lemma 2.23. Let $\mathfrak{F}=(W, R, E)$ be a descriptive MS4-frame. For each closed $A \subseteq W$ we have $A \subseteq$ $R^{-1} \mathrm{qmax} A$.

\section{Definition 2.24.}

(1) The monadic Grz is the least monadic extension MGrz of Grzegorczyk's logic Grz.

(2) An MGrz-algebra is an mm-algebra $(B, \square, \forall)$ such that $(B, \square)$ is a Grz-algebra.

(3) An MGrz-frame is an augmented Kripke frame $\mathfrak{F}=(W, R, E)$ such that $(W, R)$ is a Grz-frame.

(4) A descriptive MGrz-frame is a descriptive S4-frame $\mathfrak{F}=(W, R, E)$ validating Grzegorczyk's axiom grz.

Again, we have the following standard completeness results:

\section{Theorem 2.25.}

(1) $M G r z \vdash \varphi \Leftrightarrow \mathfrak{B} \vDash \varphi$ for each MGrz-algebra $\mathfrak{B}$.

(2) MGrz $\vdash \varphi \Leftrightarrow \mathfrak{F} \vDash \varphi$ for each descriptive MGrz-frame $\mathfrak{F}$.

It is well known that an S4-frame $\mathfrak{F}=(W, R)$ is a Grz-frame iff $R$ is a Noetherian partial order; that is, a partial order with no infinite ascending chains (of distinct points). Thus, if $\mathfrak{F}$ is finite, then $\mathfrak{F}$ is a Grz-frame iff $R$ is a partial order.

It is a result of Esakia that a descriptive S4-frame $\mathfrak{F}=(W, R)$ is a descriptive Grz-frame iff for each clopen $A \subseteq W$ the $R$-maximal and quasi- $R$-maximal points of $A$ coincide. These results clearly hold for MGrz as well.

Lemma 2.26 ([13]).

(1) Let $\mathfrak{F}=(W, R, E)$ be a descriptive MS4-frame. Then $\mathfrak{F} \vDash \operatorname{grz}$ iff for each clopen $A$ we have qmax $A=$ $\max A$.

(2) Let $\mathfrak{F}=(W, R, E)$ be a descriptive MGrz-frame. For each clopen $A$ we have $A \subseteq R^{-1} \max A$.

\section{Definition 2.27.}

(1) The monadic GL is the least monadic extension MGL of the Gödel-Löb logic GL. 
(2) An MGL-algebra is an mm-algebra $(B, \square, \forall)$ such that $(B, \square)$ is a $\mathrm{GL}$-algebra.

(3) An MGL-frame is an augmented Kripke frame $\mathfrak{F}=(W, R, E)$ such that $(W, R)$ is a GL-frame.

(4) A descriptive $\mathrm{MGL}$-frame is a descriptive augmented Kripke frame $\mathfrak{F}=(W, R, E)$ validating $\mathrm{gl}$.

As before, we have the following standard completeness results:

Theorem 2.28.

(1) MGL $\vdash \varphi \Leftrightarrow \mathfrak{B} \vDash \varphi$ for each MGL-algebra $\mathfrak{B}$.

(2) MGL $\vdash \varphi \Leftrightarrow \mathfrak{F} \vDash \varphi$ for each descriptive MGL-frame $\mathfrak{F}$.

It is well known that a Kripke frame $\mathfrak{F}=(W, R)$ is a GL-frame iff $R$ is transitive and dually well founded (no infinite ascending chains). Call $R$ a strict partial order if $R$ is irreflexive, antisymmetric, and transitive. If $W$ is finite, then $\mathfrak{F}$ is a GL-frame iff $R$ is a strict partial order.

A characterization of descriptive GL-frames was originally established by Esakia and given in [1]. It generalizes directly to descriptive MGL-frames. For a transitive frame $\mathfrak{F}=(W, R)$ and $A \subseteq W$, define the irreflexive maximum of $A$ by

$$
\mu(A)=\{x \in A \mid R(x) \cap A=\varnothing\} .
$$

Lemma $2.29([1])$. Let $\mathfrak{F}=(W, R, E)$ be a descriptive augmented Kripke frame. Then $\mathfrak{F}$ is a descriptive MGL-frame iff $\mathfrak{F}$ is transitive and $A \subseteq \mu(A) \cup R^{-1} \mu(A)$ for each clopen $A$.

Thus, a descriptive augmented Kripke frame is a descriptive MGL-frame iff it is transitive and each point in a clopen set is either in the irreflexive maximum of the clopen or sees a point in the irreflexive maximum. It was observed by Japaridze [23, 24] that MGL has the finite model property.

Theorem 2.30 (Japaridze). MGL $\vdash \varphi \Leftrightarrow \mathfrak{F} \vDash \varphi$ for all finite MGL-frames $\mathfrak{F}$.

\section{The Gödel and Splitting translations in the MONADiC SETting}

In this section we discuss the Gödel and splitting translations in the monadic setting. While the Gödel translation embeds MIPC fully and faithfully into MGrz, the splitting translation from MGrz into MGL does not yield a faithful embedding.

3.1. Gödel translation. The Gödel translation extends to the monadic setting by defining

$$
\begin{aligned}
& (\forall \varphi)^{t}=\square \forall \varphi^{t} \\
& (\exists \varphi)^{t}=\exists \varphi^{t} .
\end{aligned}
$$

Using algebraic semantics, Fisher-Servi [17, 18, proved that this provides a full and faithful embedding of MIPC into MS4. The proof also yields a full and faithful embedding of MIPC into MGrz. Below we give an alternate proof of this result, using relational semantics. The proof extends a semantical proof that IPC $\vdash \varphi$ iff S4 $\vdash \varphi^{t}$ as given, e.g., in [10, pp. 96-97].

For notational simplicity, we abbreviate the formula $\square \forall \psi$ as $\mathbf{\square}$ and the formula $\diamond \exists \psi$ as $\downarrow \psi$. Observe that this keeps the duality between box and diamond since $\psi=\neg \neg \psi$ as $\psi=\square \forall \psi=\neg \diamond \neg \neg \exists \neg \psi$, which is provably equivalent to $\neg \diamond \exists \neg \psi=\neg \checkmark \neg \psi$.

Remark 3.1. The modalities $\square$ are S4-modalities which can be modeled using the relation $Q=R \circ E$, i.e., we have

$$
\begin{array}{lll}
w \vDash \square & \text { iff } & \text { (for all } v)(w Q v \text { implies } v \vDash \varphi) ; \\
w \vDash & \text { iff } & \text { (there exists } v)(w Q v \text { and } v \vDash \varphi) .
\end{array}
$$

Using this notation, the $\forall$-step in the Gödel translation is

$$
(\forall \varphi)^{t}=\boldsymbol{\nabla} \varphi^{t} .
$$

For an MS4-frame $\mathfrak{F}=(W, R, E)$ define an equivalence relation $\sim$ on $\mathfrak{F}$ by

$$
x \sim y \text { iff } x R y \text { and } y R x .
$$


Let $[x]$ denote the equivalence class of $x$, and let $W_{\sim}=W / \sim$ be the set of all equivalence classes. Define $R_{\sim}$ and $E_{\sim}$ on $W_{\sim}$ by

$$
\begin{array}{lll}
{[x] R_{\sim}[y]} & \text { iff } & x R y ; \\
{[x] E_{\sim}[y]} & \text { iff } & x Q y \text { and } y Q x .
\end{array}
$$

That $E_{\sim}$ is well defined follows from $R \circ Q \circ R \subseteq Q$ which is true by commutativity in $\mathfrak{F}$ and transitivity of $R$. Let $\mathfrak{F}_{\sim}=\left(W_{\sim}, R_{\sim}, E_{\sim}\right)$. Set $Q_{\sim}=E_{\sim} \circ R_{\sim}$.

Lemma 3.2. Let $\mathfrak{F}=(W, R, E)$ be an MS4-frame and $x, y \in W$.

(1) $x$ Ey implies $[x] E_{\sim}[y]$;

(2) $x Q y$ iff $[x] Q \sim[y]$.

Proof. (1) If $x E y$, then $x Q y$ and $y Q x$, so $[x] E_{\sim}[y]$ by definition of $E_{\sim}$.

(2) Suppose that $x Q y$. Then there is $y^{\prime}$ with $x R y^{\prime}$ and $y^{\prime} E y$. Therefore, $[x] R\left[y^{\prime}\right]$ by definition of $R$ and $\left[y^{\prime}\right] E_{\sim}[y]$ by (1). Thus, $[x] Q_{\sim}[y]$. Conversely, if $[x] Q_{\sim}[y]$, then there is $\left[y^{\prime}\right]$ with $[x] R_{\sim}\left[y^{\prime}\right]$ and $\left[y^{\prime}\right] E_{\sim}[x]$. By the definitions of $R_{\sim}$ and $E_{\sim}$, we have $x R y^{\prime}$ and $y^{\prime} Q y$. Thus, $x Q y$.

Lemma 3.3. $\mathfrak{F}_{\sim}$ is an MIPC-frame.

Proof. It is well known (and easy to verify) that $R_{\sim}$ is a partial order (see, e.g., [10, p. 68]). Transitivity and reflexivity of $E_{\sim}$ easily follow from transitivity and reflexivity of $Q$, and $E_{\sim}$ is symmetric by definition. To see that $\mathfrak{F}_{\sim}$ satisfies commutativity, let $[x],[y],[z] \in W_{\sim}$ with $[x] E_{\sim}[y]$ and $[y] R_{\sim}[z]$. Then $x Q y$ and $y R z$, so $x Q z$. Therefore, there is $z^{\prime}$ with $x R z^{\prime}$ and $z^{\prime} E z$. From $x R z^{\prime}$ it follows that $[x] R\left[z^{\prime}\right]$, and $z^{\prime} E z$ implies $\left[z^{\prime}\right] E_{\sim}[z]$ by Lemma $3.2(1)$. Thus, $\mathfrak{F}_{\sim}$ satisfies commutativity.

Given a valuation $\nu$ on $\mathfrak{F}$, define a valuation $\nu_{\sim}$ on $\mathfrak{F} \sim$ by

$$
\nu \sim(p)=\{[x] \mid x \in \nu(\square p)\} .
$$

Clearly $\nu_{\sim}(p)$ is an upset. We call $\mathfrak{F}_{\sim}$ the skeleton of $\mathfrak{F}$ and $\left(\mathfrak{F}_{\sim}, \nu_{\sim}\right)$ the skeleton of $(\mathfrak{F}, \nu)$.

Conversely, given an MIPC-frame $\mathfrak{F}$, we regard it as an MS4-frame. In addition, if $\mathfrak{F}$ is finite, then we regard it as a finite MGrz-frame. If $\nu$ is a valuation on the MIPC-frame $\mathfrak{F}$, then we regard it as a valuation on the MGrz-frame $\mathfrak{F}$.

The following lemma describes how the above frame transformations behave with respect to the Gödel translation. It is proved by induction on the complexity of $\varphi$.

Lemma 3.4. Let $\varphi$ be a formula of $\mathcal{L}_{\forall \exists}$.

(1) For an MIPC-frame $\mathfrak{F}$ with a valuation $\nu$ and $x \in \mathfrak{F}$ we have

$$
(\mathfrak{F}, x) \vDash_{\nu} \varphi \Leftrightarrow(\mathfrak{F}, x) \vDash_{\nu} \varphi^{t} .
$$

(2) For an MS4-frame $\mathfrak{F}$ with a valuation $\nu$ and $x \in \mathfrak{F}$, we have

$$
(\mathfrak{F}, x) \vDash_{\nu} \varphi^{t} \Leftrightarrow\left(\mathfrak{F}_{\sim},[x]\right) \models_{\nu_{\sim}} \varphi .
$$

Proof. If $\mathfrak{F}$ is an MIPC-frame, then $\mathfrak{F}_{\sim}$ is isomorphic to $\mathfrak{F}$. Therefore, (1) follows from (2). To prove (2), by [10, Lem. 3.81], it is sufficient to only consider the case for the modalities $\forall$ and $\exists$. Let $\varphi=\forall \psi$. Then

$$
\begin{aligned}
{[x] \vDash \forall \psi } & \Leftrightarrow \quad(\text { for all }[y])\left([x] Q_{\sim}[y] \Rightarrow[y] \vDash \psi\right) \\
& \Leftrightarrow \quad(\text { for all }[y])\left([x] Q_{\sim}[y] \Rightarrow y \vDash \psi^{t}\right) \quad \text { (Inductive Hypothesis) } \\
& \Leftrightarrow \quad(\text { for all } y)\left(x Q y \Rightarrow y \vDash \psi^{t}\right) \quad(\text { Lemma } 3.2(2)) \\
& \Leftrightarrow x \vDash \mathbf{\square} \psi^{t} \\
& \Leftrightarrow \vDash(\forall \psi)^{t} .
\end{aligned}
$$

Next let $\varphi=\exists \psi$. If $x \models(\exists \psi)^{t}$, then there is $y$ with $x E y$ and $y \models \psi^{t}$. Therefore, $[y] \models \psi$ by the inductive hypothesis, and $[x] E[y]$ by Lemma $3.2(1)$. Thus, $[x] \models \exists \psi$. Conversely, suppose that $[x] \models \exists \psi$. Then there is $[y]$ with $[x] E_{\sim}[y]$ and $[y] \models \psi$. Therefore, $y Q x$ by the definition of $E_{\sim}$. Thus, there is $x^{\prime}$ with $y R x^{\prime}$ and $x^{\prime} E x$. By the definition of $R_{\sim}$, we have $[y] R_{\sim}\left[x^{\prime}\right]$. So $\left[x^{\prime}\right] \models \psi$ by the persistence in $\mathfrak{F}_{\sim}$. Consequently, $x^{\prime} \models \psi^{t}$ by the inductive hypothesis, and hence $x \models \exists \psi^{t}=(\exists \psi)^{t}$.

Theorem 3.5. MIPC $\vdash \varphi$ iff MS4 $\vdash \varphi^{t}$ iff MGrz $\vdash \varphi^{t}$. 
Proof. Suppose that MIPC $\forall \varphi$. Since MIPC has the FMP (Theorem 2.10), there exists a finite MIPC-frame $\mathfrak{F}$, a valuation $\nu$ on $\mathfrak{F}$, and $x \in \mathfrak{F}$ such that $x \not{ }_{\nu} \varphi$. By regarding $\mathfrak{F}$ as an MGrz-frame, $x \not \forall_{\nu} \varphi^{t}$ by Lemma 3.4(1). Therefore, MGrz $\forall \varphi^{t}$. Also, as MS4 $\subseteq$ MGrz, it follows that MS4 $\forall \varphi^{t}$.

Conversely, if MGrz $\forall \varphi^{t}$, then MS4 $\forall \varphi^{t}$. By the FMP for MS4, there is a finite MS4-frame $\mathfrak{F}$, a valuation $\nu$ on $\mathfrak{F}$, and $x \in \mathfrak{F}$ such that $(\mathfrak{F}, x) \not \nvdash_{\nu} \varphi^{t}$. By Lemma $3.4(2),\left(\mathfrak{F}_{\sim},[x]\right) \not \nu_{\nu} \varphi$. Thus, MIPC $\forall \varphi$.

3.2. Splitting translation. Next we discuss the splitting translation in the monadic setting. The key here is Esakia's observation that the splitting translation does not yield a faithful embedding of MGrz into MGL. Since this result is unpublished, we give a proof of it.

Definition 3.6. Let $\mathfrak{F}=(W, R, E)$ be an augmented Kripke frame (modal or intuitionistic), and let $x \in W$.

(1) An E-cluster (or cluster) is a subset of $W$ of the form $E(x)=\{w \in W: x E w\}$ (it is the equivalence class of $x \in W$ with respect to $E$ ).

(2) We say that the $E$-cluster $E(x)$ is dirty if there are $u, v \in E(x)$ with $u \neq v$ and $u R v$.

(3) We say that the cluster is clean otherwise; that is, $u, v \in E(x)$ and $u R v$ imply $u=v$.

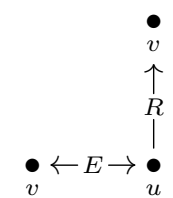

Dirty cluster

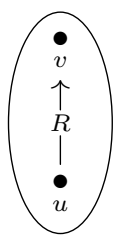

Dirty cluster (alternate depiction - oval represents $E$-cluster)

Descriptive MGL-frames have the property that clusters in the irreflexive maximum of an $E$-saturated clopen are clean.

Lemma 3.7. Let $\mathfrak{F}=(W, R, E)$ be a descriptive MGL-frame. For clopen $A$ and $m \in \mu(E(A))$ we have that $E(m)$ is clean.

Proof. Suppose there exist clopen $A$ and $m \in \mu(E(A))$ with $E(m)$ dirty. Then there are $x, y \in E(m)$ with $x R y, x E y$, and $x \neq y$. By commutativity, there is $w$ such that $m R w$ and $w E y$, as shown below.

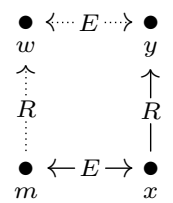

Since $y \in E(A)$ we have $w \in E(A)$. But this contradicts $R(m) \cap E(A)=\varnothing$. Thus, we cannot have a dirty cluster in $\mu(E(A))$.

As a consequence of Lemma 3.7, we obtain:

Lemma 3.8. Finite MGL-frames are finite strict partial orders in which all clusters are clean.

We next show that the splitting of the Gödel translation of the monadic version of Casari's formula

$$
\text { MCas : } \forall((p \rightarrow \forall p) \rightarrow \forall p) \rightarrow \forall p
$$

is provable in MGL.

Since $\square \forall p \leftrightarrow \square \forall \square p$ is provable in MS4, it is straightforward to check that (MCas) ${ }^{t}$ is provably equivalent to $\square \forall(\square(\square p \rightarrow \square \forall p) \rightarrow \square \forall p) \rightarrow \square \forall p$. Using the notation $\square$ introduced above, we have that (MCas) ${ }^{t}$ is:

$$
\mathrm{M}_{\square} \text { Cas : } \quad \mathbf{\square}(\square(\square p \rightarrow \mathbf{\square} p) \rightarrow \mathbf{\square} p) \rightarrow \mathbf{\square} p .
$$

Note that $(\mathbf{\square} \psi)^{s}=(\square \forall \psi)^{s}=\square^{+} \forall \psi=\forall \psi \wedge \square \forall \psi=\forall \psi \wedge \boldsymbol{\square} \psi$. So we can use $\mathbf{\square}^{+} \psi$ to abbreviate $\forall \psi \wedge \square \forall \psi=\square^{+} \forall \psi$, and so the splitting translation of $\mathrm{M}_{\square}$ Cas is

$$
\left(\mathrm{M}_{\square} \mathrm{Cas}\right)^{s}=\mathbf{\square}^{+}\left(\square^{+}\left(\square^{+} p \rightarrow \mathbf{\square}^{+} p\right) \rightarrow \mathbf{\square}^{+} p\right) \rightarrow \mathbf{\square}^{+} p .
$$


Theorem 3.9. $\mathrm{MGL} \vdash\left(\mathrm{M}_{\square} \mathrm{Cas}\right)^{s}$.

Proof. Suppose $\mathfrak{F}=(W, R, E)$ is a descriptive $\mathrm{MGL}$-frame. We will prove that $\mathfrak{F} \vDash\left(\mathrm{M}_{\square} \mathrm{Cas}\right)^{s}$. Let $\nu$ be a valuation on $\mathfrak{F}, x \in \mathfrak{F}$, and $x \not \forall_{\nu} \mathbf{\square}^{+} p$. We show that $x \not \forall \mathbf{\square}^{+}\left(\square^{+}\left(\square^{+} p \rightarrow \mathbf{\square}^{+} p\right) \rightarrow \mathbf{\square}^{+} p\right)$. Let $A=W \backslash \nu\left(\boldsymbol{\square}^{+} p\right)$. Then $x \in A$ and so by Lemma 2.29, $x \in \mu(A) \cup R^{-1} \mu(A)$. If $x \in R^{-1} \mu(A)$, then there is $x^{\prime} \in \mu(A)$ with $x R x^{\prime}$. If $x \in \mu(A)$, we let $x^{\prime}=x$. From $x^{\prime} \in \mu(A)$ it follows that $x^{\prime} \in A$, so $x^{\prime} \not \forall \mathbf{\square}^{+} p=\forall p \wedge \square \forall p$. We show that $x^{\prime} \not \forall \forall p$. If $x^{\prime} \not \forall \square \forall p$, then there is $y$ with $x^{\prime} R y$ and $y \not \forall \forall p$. Therefore, $y \not \forall \mathbf{\square}^{+} p$, so $y \in A$. But this contradicts $x^{\prime} \in \mu(A)$. Thus, $x^{\prime} \not \forall \forall p$. So there is $w$ with $w E x^{\prime}$ and $w \not \models p$. We show that $w \not \models \square^{+}\left(\square^{+} p \rightarrow \mathbf{\square}^{+} p\right) \rightarrow \mathbf{\square}^{+} p$.

Since $w \not \forall p$, we have $w \not \models p \wedge \square p$, so $w \not \models \square^{+} p$, and hence $w \vDash \square^{+} p \rightarrow \mathbf{\square}^{+} p$. Let $w R z$. By commutativity, there is $y$ such that $x^{\prime} R y$ and $y E z$. Since $x^{\prime} \in \mu(A)$, we have $y \notin A$. Therefore, $y \vDash \mathbf{\square}^{+} p$, so $y \vDash \forall p$, and hence $z \vDash \forall p$.

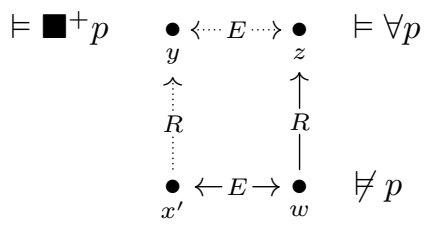

In fact, if $z R t$, then $w R t$ by transitivity, and so by the same reasoning as above we have $t \vDash \forall p$. It follows that $z \vDash \square \forall p$, and so $z \vDash \mathbf{\square}^{+} p$. Thus, $z \vDash \square^{+} p \rightarrow \mathbf{\square}^{+} p$, and hence $w \vDash \square\left(\square^{+} p \rightarrow \mathbf{\square}^{+} p\right)$. This together with $w \vDash \square^{+} p \rightarrow \boldsymbol{\square}^{+} p$ yields $w \vDash \square^{+}\left(\square^{+} p \rightarrow \mathbf{\square}^{+} p\right)$. Since $w \not \models \boldsymbol{\square}^{+} p$, we obtain $w \not \square^{+}\left(\square^{+} p \rightarrow \mathbf{\square}^{+} p\right) \rightarrow \boldsymbol{\square}^{+} p$.

If $x=x^{\prime}$, then $x E w$, and so $x \not \forall \forall\left(\square^{+}\left(\square^{+} p \rightarrow \mathbf{\square}^{+} p\right) \rightarrow \mathbf{\square}^{+} p\right)$. Otherwise, $x R x^{\prime}$ and $x^{\prime} E w$ imply $x Q w$, so $x \not=\boldsymbol{\square}\left(\square^{+}\left(\square^{+} p \rightarrow \boldsymbol{\square}^{+} p\right) \rightarrow \boldsymbol{\square}^{+} p\right)$. Thus, in either case, $x \not \models \mathbf{\square}^{+}\left(\square^{+}\left(\square^{+} p \rightarrow \boldsymbol{\square}^{+} p\right) \rightarrow \boldsymbol{\square}^{+} p\right)$ as desired. This yields $x \vDash\left(\mathrm{M}_{\square} \mathrm{Cas}\right)^{s}$. Since $x$ was arbitrary, $\mathfrak{F} \vDash\left(\mathrm{M}_{\square} \text { Cas }\right)^{s}$. Because $\mathfrak{F}$ is an arbitrary descriptive MGL-frame, by Theorem 2.28(2), MGL $\vdash\left(\mathrm{M}_{\square} \mathrm{Cas}\right)^{s}$.

Theorem 3.10. $M G r z \not \forall M_{\square}$ Cas.

Proof. Consider the MGrz-frame $\mathfrak{F}=(W, R, E)$ where $W=\{x, y\}, R=\{(x, x),(y, y),(x, y)\}$, and $E=$ $W^{2}=\{(x, x),(y, y),(x, y),(y, x)\}$, as shown below.

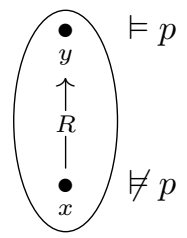

The arrow represents the nontrivial $R$-relation and the circle represents that both points are in the same $E$-equivalence class. It is easy to see that this is an MGrz-frame. Let $\nu$ be a valuation on $\mathfrak{F}$ with $\nu(p)=\{y\}$.

First, we claim that $x \vDash \mathbf{\square}(\square(\square p \rightarrow \square p) \rightarrow \mathbf{\square})$. To see this, note that both $x \not \forall \mathbf{\square}$ and $y \not \forall \mathbf{\square} p$, but since $y \vDash p$ and $y$ only sees itself (with respect to $R$ ), we have $y \vDash \square p$. Thus, $y \not \forall \square p \rightarrow \square p$, so $x \not \models \square(\square p \rightarrow \square p)$, and hence $x \vDash \square(\square p \rightarrow \square p) \rightarrow \square$. Moreover, $y \not \forall \square(\square p \rightarrow \square p)$, so $y \vDash \square(\square p \rightarrow \square p) \rightarrow \square p$, and hence $x \vDash \mathbf{\square}(\square(\square p \rightarrow \square p) \rightarrow \square p)$. However, $x \not=\square p$ as $x Q x$ and $x \not p p$. Thus, $x \not \forall \mathbf{\square}(\square(\square p \rightarrow \square p) \rightarrow \square p) \rightarrow \square p$, hence $\mathfrak{F} \forall_{M G r z} M_{\square}$ Cas, and so $M G r z \forall M_{\square}$ Cas.

Corollary 3.11. (Esakia) The splitting translation does not embed MGrz into MGL faithfully.

\section{The LOGICS $\mathrm{M}^{+}$IPC AND $\mathrm{M}^{+} \mathrm{Grz}$}

In the previous section we saw that the splitting translation does not embed MGrz into MGL faithfully. In fact, while the Gödel translation of MCas is not provable in MGrz, the splitting translation of the Gödel translation of MCas is provable in MGL. Esakia suggested to strengthen MIPC with MCas and MGrz with the Gödel translation of MCas, and see whether this repairs the disbalance. This is what we do in this section. 


\section{1. $\mathrm{M}^{+} \mathrm{IPC}$.}

Definition 4.1. The logic $M^{+} I P C$ is defined as the extension of MIPC by MCas:

$$
\mathrm{M}^{+} \mathrm{IPC}=\mathrm{MIPC}+\mathrm{MCas} \text {. }
$$

Recall from Definition 3.6 that a cluster of an MIPC-frame is called clean if no distinct points in the cluster are $R$-related. The following semantic characterization of $\mathrm{M}^{+} \mathrm{IPC}$-frames was established by Esakia. For a proof see [4, Lem. 38]. It states that a descriptive MIPC-frame is a descriptive $\mathrm{M}^{+}$IPC-frame iff the cluster of each point in the $R$-maximum of the $E$-saturation of clopen is clean.

Lemma 4.2. [4, Lem. 38] Let $\mathfrak{F}=(W, R, E)$ be a descriptive MIPC-frame. Then $\mathfrak{F} \vDash M C a s$ iff for each clopen $A$, if $m \in \max E(A)$, then $E(m)$ is clean.

Remark 4.3. The condition in [4, Lem. 38] is that $\mathfrak{F} \vDash M C$ as iff for each clopen $A$ we have $A \subseteq Q^{-1}(\max A \cap$ $\max Q^{-1} A$ ). But, as discussed after the proof of [4, Lem. 38], this statement is equivalent to the statement in Lemma 4.2 .

As a consequence of Lemma 4.2, we obtain:

Lemma 4.4. Finite $\mathrm{M}^{+} \mathrm{IPC}$-frames are finite MIPC-frames in which all clusters are clean.

4.2. $\mathrm{M}^{+} \mathrm{Grz}$.

Definition 4.5. The logic $\mathrm{M}^{+} \mathrm{Grz}$ is the extension of $M G r z$ by $\mathrm{M}_{\square}$ Cas:

$$
\mathrm{M}^{+} \mathrm{Grz}=\mathrm{MGrz}+\mathrm{M}_{\square} \text { Cas. }
$$

Remark 4.6. As we pointed out in the previous section, $M_{\square}$ Cas is provably equivalent to the Gödel translation of MCas.

In order to obtain a semantic characterization of $\mathrm{M}^{+} \mathrm{Grz}$, which is an analogue of Lemma 4.2 , we require the following lemma.

Lemma 4.7. Let $\mathfrak{F}=(W, R, E)$ be a descriptive MGrz-frame, $A \subseteq W$ clopen, and $y \in \max E(A)$. If $E(y)$ is clean, then:

(1) $E(y) \subseteq \max E(A)$;

(2) for all $z \in W$, from $y R z$ and $z R y$ it follows that $y=z$.

Proof. (1) Let $z \in E(y)$ and $w \in E(A)$ with $z R w$. By commutativity, there is $w^{\prime}$ with $y R w^{\prime}$ and $w^{\prime} E w$. Therefore, $w^{\prime} \in E(A)$. Since $y \in \max E(A)$, we have $y=w^{\prime}$. Thus, $z, w \in E(y)$ and $z R w$. As $E(y)$ is clean, $z=w$. This shows that $z \in \max E(A)$.

(2) Suppose $y R z$ and $z R y$. From $y \in E(A)$ and $y R y$, we have $y \in R^{-1} E(A)$. We show that $y \in$ qmax $R^{-1} E(A)$. Let $y R w$ and $w \in R^{-1} E(A)$, so $w R u$ for some $u \in E(A)$. Then $y R u$ by transitivity, and $y \in \max E(A)$ implies $y=u$, hence $w R y$, and so $y \in \operatorname{qmax} R^{-1} E(A)$. By Lemma 2.26(1), this means $y \in \max R^{-1} E(A)$. Since $z R y$, we have $z \in R^{-1} E(A)$, so $y R z$ implies $z=y$.

We now have the necessary machinery to prove a semantic characterization of $\mathrm{M}^{+} \mathrm{Grz}$, which states that a descriptive $M G r z$-frame is a descriptive $\mathrm{M}^{+} \mathrm{Grz}$-frame iff the cluster of every point in the maximum of the $E$-saturation of a clopen set is clean.

Lemma 4.8. Let $\mathfrak{F}=(W, R, E)$ be a descriptive MGrz-frame. Then $\mathfrak{F} \vDash \mathrm{M}_{\square}$ Cas iff for each clopen $A$ and $m \in \max E(A)$ we have $E(m)$ is clean.

Proof. First suppose $\mathfrak{F} \not \mid \mathrm{M}_{\square}$ Cas. Then there is $x \in W$ such that $x \not \forall \mathbf{\square}(\square(\square p \rightarrow \mathbf{\square} p) \rightarrow \mathbf{\square} p) \rightarrow \mathbf{\square}$, and hence $x \vDash \mathbf{\square}(\square(\square p \rightarrow \square p) \rightarrow \square p)$ but $x \not \models \square p$. Since $x \not \forall \square p$, there is $x^{\prime} \in W$ such that $x Q x^{\prime}$ and $x^{\prime} \not p p$. Let $A=\{w \in W|w \not| p\}$. Then $x^{\prime} \in A$, and as $x^{\prime} E x^{\prime}$, we have $x^{\prime} \in E(A)$. Because $A$ is clopen, so is $E(A)$. By Lemma 2.26(2), there is $y \in \max E(A)$ with $x^{\prime} R y$. If $E(y)$ is dirty, then we are done. So assume that $E(y)$ is clean. We show that this leads to a contradiction. Since $y \in E(A)$, there is $y^{\prime} \in A$ with $y E y^{\prime}$. By Lemma 4.7(1), $y^{\prime} \in \max E(A)$. Because $x Q y^{\prime}$ and $x \vDash \mathbf{\square}(\square(\square p \rightarrow \square p) \rightarrow \square p)$, we have $y^{\prime} \vDash \square(\square p \rightarrow \square p) \rightarrow \square p$. As $y^{\prime} \in A$, we have $y^{\prime} \not \models p$ and since $y^{\prime} Q y^{\prime}$, we have $y^{\prime} \not \forall \square p$, so we must have $y^{\prime} \not \forall \square(\square p \rightarrow \square p)$. Thus, there is $z \in W$ such that $y^{\prime} R z$ and $z \not \models \square p \rightarrow \square p$, which means $z \vDash \square p$ but $z \not \forall \square p$. Because $z \not=\square p$, there exist $w^{\prime}, w \in W$ such that $z R w^{\prime} E w$ and $w \not \models p$ (see the diagram below). 


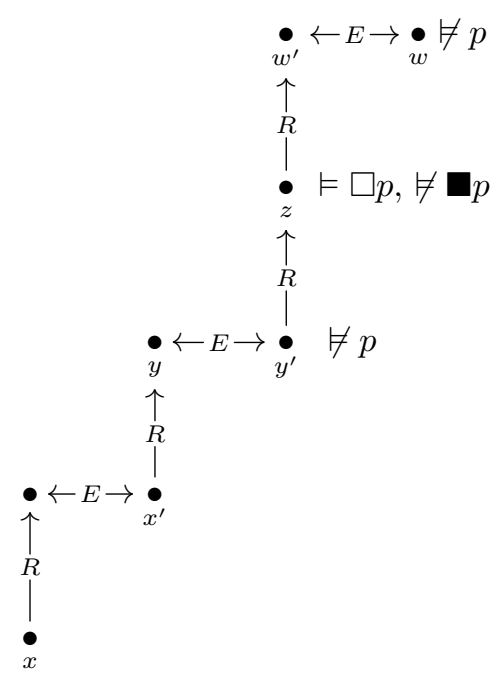

Now, since $w \not \not p$, we have $w \in A$ and hence $w^{\prime} \in E(A)$. Thus, $y^{\prime} R w^{\prime}$ and $w^{\prime} \in E(A)$, so by $R$-maximality of $y^{\prime}$ in $E(A)$, we must have $y^{\prime}=w^{\prime}$. But then $y^{\prime} R z$ and $z R y^{\prime}$, and so by Lemma 4.7 (2), $y^{\prime}=z$. This, however, is a contradiction since $z \vDash \square p$, hence $z \vDash p$, whereas $y^{\prime} \not \models p$.

For the converse, suppose that $A$ is clopen and $m \in \max E(A)$ with $E(m)$ dirty. First observe that since $m$ is maximal in $E(A)$, from $m Q t$ it follows that $t \in E(m)$ for all $t \in E(A)$. Indeed, if $m Q t$ for $t \in E(A)$, then there is $t^{\prime}$ with $m R t^{\prime}$ and $t^{\prime} E t$. Since $t^{\prime} \in E(A)$, we have $t^{\prime}=m$ by maximality of $m$ in $E(A)$. Thus, $t \in E(m)$.

Now, since $E(m)$ is dirty, there are $x, x^{\prime} \in E(m)$ with $x R x^{\prime}$ and $x \neq x^{\prime}$. In particular, $x \notin \max E(A)$. Since $E(A)$ is clopen, $\max E(A)$ is closed (see, e.g., [14, Sec. III.2]). Thus, we can find clopen $B$ such that $x \in B$ but $B \cap \max E(A)=\varnothing$, as shown below.

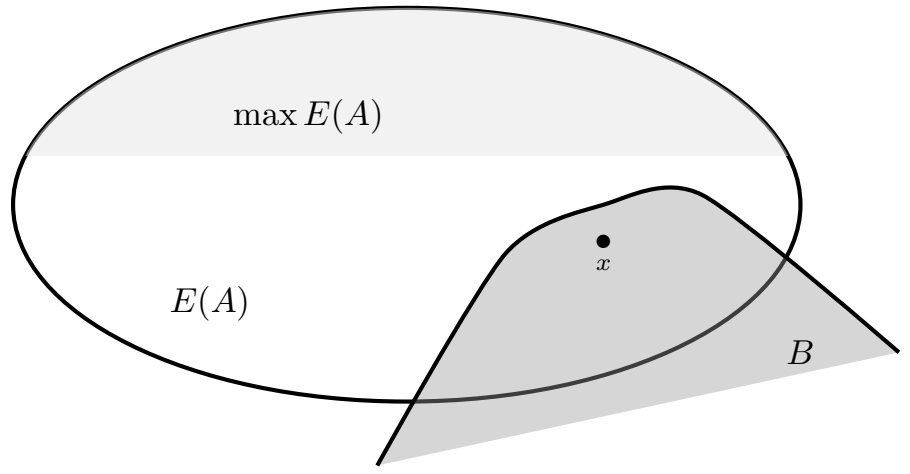

Choose a valuation $\nu$ with $\nu(p)=W \backslash(B \cap E(A))$. Note that $\nu$ is well-defined as $B$ and $E(A)$ are clopen. We aim to show that $x \vDash \mathbf{\square}(\square(\square p \rightarrow \square p) \rightarrow \square p)$ but $x \not \models \square p$. Since $x \in B \cap E(A)$, we have $x \not \models p$. This implies that $x \not \forall \mathbf{\square}$ because $x Q x$. To finish the argument it suffices to show that $y=\square(\square p \rightarrow \mathbf{\square}) \rightarrow \mathbf{\square} p$ for all $y$ with $x Q y$. So let $x Q y$ and assume that $y \not \models \mathbf{\square}$. Then there is $z$ with $y Q z$ and $z \not \neq p$. Therefore, $z \in B \cap E(A)$ and there is $z^{\prime}$ with $y R z^{\prime}$ and $z^{\prime} E z$. Clearly $z^{\prime} \in E(A)$. By Lemma 2.26(2), there is $t \in \max E(A)$ with $z^{\prime} R t$. 


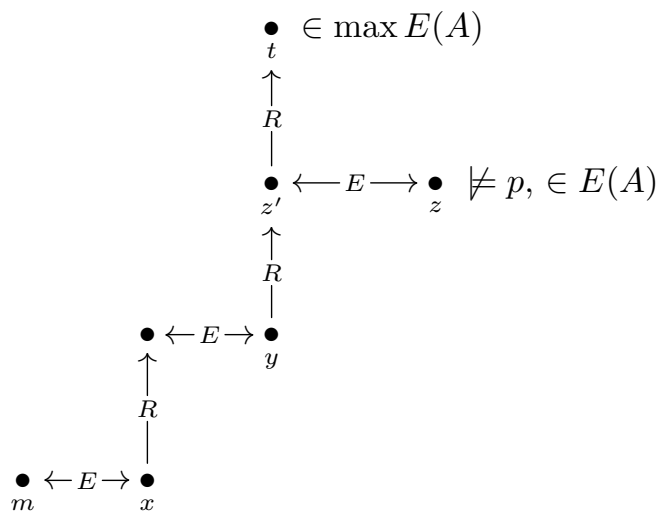

Since $t \in \max E(A)$, we have $t \notin B$, so $t=p$ and if $t R v$ for $t \neq v$, then $v \notin E(A)$ by maximality of $t$, so $v \models p$. Thus, $t \models \square p$. On the other hand, $x Q y, y R z^{\prime}$, and $z^{\prime} R t$ imply $m Q t$. As we saw above, this means $t \in E(m)$, and so $t E x$. Since $x \not \models p$, we have $t \not \models \square p$. This implies that $t \not \models \square p \rightarrow \square p$, so $y \not \models \square(\square p \rightarrow \square p)$, and hence $y \models \square(\square p \rightarrow \mathbf{\square} p) \rightarrow \mathbf{\square} p$ as desired.

As a consequence of Lemma 4.8, we obtain:

Lemma 4.9. Finite $\mathrm{M}^{+} \mathrm{Grz}$-frames are finite $\mathrm{MGrz-frames} \mathrm{in} \mathrm{which} \mathrm{all} \mathrm{clusters} \mathrm{are} \mathrm{clean.}$

4.3. The translations $\mathrm{M}^{+} \mathrm{IPC} \rightarrow \mathrm{M}^{+} \mathrm{Grz} \rightarrow \mathrm{MGL}$. As we pointed out, the remaining part of the paper establishes the finite model property for the logics $\mathrm{M}^{+} \mathrm{IPC}$ and $\mathrm{M}^{+} \mathrm{Grz}$. We finish this section by explaining how a proof of Esakia's claim is then obtained.

Let $R$ be a binary relation. We recall that the irreflexive reduction of $R$, denoted $R^{i}$, is defined by

$$
a R^{i} b \text { iff } a R b \text { and } a \neq b \text {; }
$$

and the reflexive closure of $R$, denoted $R^{r}$, is defined by

$$
a R^{r} b \text { iff } a R b \text { or } a=b .
$$

For an augmented Kripke frame $\mathfrak{F}=(W, R, E)$, let $\mathfrak{F}^{i}=\left(W, R^{i}, E\right)$ and $\mathfrak{F}^{r}=\left(W, R^{r}, E\right)$. Following the terminology of [10, pp. 98-99], we call $\mathfrak{F}^{i}$ the irreflexive reduction and $\mathfrak{F}^{r}$ the reflexive closure of $\mathfrak{F}$.

\section{Lemma 4.10.}

(1) If $\mathfrak{F}$ is a finite $\mathrm{M}^{+} \mathrm{Grz}$-frame, then $\mathfrak{F}^{i}$ is a finite $\mathrm{MGL}$-frame.

(2) If $\mathfrak{F}$ is a finite $\mathrm{MGL}$-frame, then $\mathfrak{F}^{r}$ is a finite $\mathrm{M}^{+} \mathrm{Grz}$-frame.

Proof. Since finite $\mathrm{M}^{+}$Grz-frames are finite partial orders with clean clusters (Lemma 4.9) and finite MGLframes are finite strict partial orders with clean clusters (Lemma 3.8), this is an immediate consequence of [10, pp. 98-99].

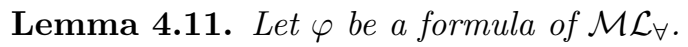

(1) For a finite $\mathrm{M}^{+} \mathrm{Grz}$-frame $\mathfrak{F}$, a valuation $\nu$ on $\mathfrak{F}$, and $x \in \mathfrak{F}$ we have

$$
(\mathfrak{F}, x) \vDash_{\nu} \varphi \Leftrightarrow\left(\mathfrak{F}^{i}, x\right) \vDash_{\nu} \varphi^{s} .
$$

(2) For a finite MGL-frame $\mathfrak{F}$, a valuation $\nu$ on $\mathfrak{F}$, and $x \in \mathfrak{F}$ we have

$$
(\mathfrak{F}, x) \vDash_{\nu} \varphi^{s} \Leftrightarrow\left(\mathfrak{F}^{r}, x\right) \models_{\nu} \varphi .
$$

Proof. The proof is an immediate consequence of [10, pp. 98-99] since the quantifier modalities are not changed by the translation $(-)^{s}$, nor is the relation $E$ altered going from $\mathfrak{F}$ to $\mathfrak{F}^{i}$ or $\mathfrak{F}^{r}$.

Finally, we are ready to provide a proof of Esakia's claim.

Theorem 4.12. $\mathrm{M}^{+} \mathrm{IPC} \vdash \varphi$ iff $\mathrm{M}^{+} \mathrm{Grz} \vdash \varphi^{t}$ iff $\mathrm{MGL} \vdash\left(\varphi^{t}\right)^{s}$. 
Proof. The first equivalence is proved exactly as Theorem 3.5 using the fact that finite $\mathrm{M}^{+} \mathrm{IPC}$-frames and finite $\mathrm{M}^{+} \mathrm{Grz}$-frames coincide.

For the second equivalence, suppose MGL $\forall\left(\varphi^{t}\right)^{s}$. Since MGL has the FMP, there exist a finite MGL-frame $\mathfrak{F}$, a valuation $\nu$ on $\mathfrak{F}$, and $x \in \mathfrak{F}$ such that $(\mathfrak{F}, x) \not \not_{\nu}\left(\varphi^{t}\right)^{s}$. By Lemma 4.11 $(2),\left(\mathfrak{F}^{r}, x\right) \not \not_{\nu} \varphi^{t}$, and since $\mathfrak{F}^{r}$ is an $\mathrm{M}^{+} \mathrm{Grz}$-frame by Lemma 4.10(2), $\mathrm{M}^{+} \mathrm{Grz} \not \forall \varphi^{t}$. For the converse, suppose $\mathrm{M}^{+} \mathrm{Grz} \not \varphi^{t}$. Since $\mathrm{M}^{+} \mathrm{Grz}$ has the FMP, there exist a finite $\mathrm{M}^{+}$Grz-frame $\mathfrak{F}$, a valuation $\nu$ on $\mathfrak{F}$, and $x \in \mathfrak{F}$ such that $(\mathfrak{F}, x) \not{ }_{\nu} \varphi^{t}$. By Lemma 4.11 $(1),\left(\mathfrak{F}^{i}, x\right) \not \forall_{\nu}\left(\varphi^{t}\right)^{s}$, and since $\mathfrak{F}^{i}$ is an MGL-frame by Lemma 4.10(1), we conclude that $\operatorname{MGL} \nvdash\left(\varphi^{t}\right)^{s}$.

We now have succeeded in lifting the original correspondences given by Goldblatt, Boolos, Kuznetsov and Muravitsky from the propositional setting to the monadic setting, verifying Esakia's claim. Combining this with Japaridze's result of arithmetical completeness for MGL yields arithmetic interpretations of $\mathrm{M}^{+} \mathrm{IPC}$ and $\mathrm{M}^{+} \mathrm{Grz}$.

\section{The FINITE MODEL PROPERTY OF $\mathrm{M}^{+}$IPC}

This section is dedicated to the proof of the finite model property of $\mathrm{M}^{+}$IPC. We do this by modifying the selective filtration technique originally developed by Grefe [22] to prove the finite model property of Fisher Servi's intuitionistic modal logic FS. In [20, §10.3] it was used to give an alternative proof of the finite model property of MIPC.

We start by collecting some properties of descriptive $\mathrm{M}^{+}$IPC-frames that will be useful in what follows. The following lemma is the $\mathrm{M}^{+} \mathrm{IPC}$-version of Lemma 4.7(1).

Lemma 5.1. Let $\mathfrak{F}=(W, R, E)$ be a descriptive $\mathrm{M}^{+} \mathrm{IPC}$-frame, $A \subseteq W$ clopen, $y \in \max E(A)$, and $E(y)$ clean. Then $E(y) \subseteq \max E(A)$.

Proof. If $E(y) \nsubseteq \max E(A)$, then there are distinct $t \in E(y)$ and $u \in E(A)$ with $t R u$. By commutativity, there is $u^{\prime}$ with $y R u^{\prime}$ and $u^{\prime} E u$. Therefore, $u^{\prime} \in E(A)$, so by maximality of $y$ in $E(A)$ we have $y=u^{\prime}$. This implies that $t E u$, contradicting that $E(y)$ is a clean cluster.

We say a point $x$ is maximal with respect to a formula $\psi$ if $x \not \models \psi$ and for each $y$ with $x R y$ and $x \neq y$ we have $y \vDash \psi$ (that is, $x$ refutes $\psi$ and every point strictly above $x$ validates $\psi$ ).

Lemma 5.2. Let $\mathfrak{F}=(W, R, E)$ be a descriptive $\mathrm{M}^{+} \mathrm{IPC}$-frame, $t \in W$, and $\nu$ a valuation on $\mathfrak{F}$.

(1) Let $A \subseteq W$ be clopen. If $t \in E(A)$, then there is $x \in \max E(A)$ such that $t R x$ and $E(x)$ is clean.

(2) If $t \not \forall \forall \varphi$, then there is $x$ such that $t R x, x$ is maximal with respect to $\forall \varphi$, and $E(x)$ is clean.

(3) Let $A \subseteq W$ be clopen. If $t \in A$, then there is $x \in A \cap \max E(A)$ such that $t Q x$ and $E(x)$ is clean.

(4) If $t \not \models \varphi$, then there is $x$ such that $t Q x, x$ is maximal with respect to $\varphi$, and $E(x)$ is clean.

Proof. (1) Let $t \in E(A)$. By Lemma 2.12, there is $x \in \max E(A)$ such that $t R x$. By Lemma $4.2 E(x)$ is clean.

(2) Suppose that $t \not \forall \forall \varphi$. Let $A=\nu(\forall \varphi)^{c}$. Then $A$ is clopen, $E(A)=A$, and $t \in E(A)$. By (1), there is $x \in \max E(A)$ such that $t R x$ and $E(x)$ is clean. Since $E(A)=A$, it immediately follows that $x$ is maximal with respect to $\forall \varphi$.

(3) Let $t \in A$. Then $t \in E(A)$. By (1), there is $x^{\prime} \in \max E(A)$ such that $t R x^{\prime}$ and $E\left(x^{\prime}\right)$ is clean. Since $x^{\prime} \in E(A)$, there is $x \in A$ with $x^{\prime} E x$. Therefore, $t Q x$, and because $E\left(x^{\prime}\right)$ is clean, we have that $x \in \max E(A)$ by Lemma 5.1 .

(4) Suppose that $t \not \forall \varphi$. Let $A=\nu(\varphi)^{c}$. Then $A$ is clopen and $t \in A$. By (3), there is $x \in A \cap \max E(A)$ such that $t Q x$ and $E(x)$ is clean. Since $x \in A$, we also have $x \in \max A$. But the latter means that $x$ is maximal with respect to $\varphi$. Thus, $x$ is as desired.

5.1. The construction. We start with a formula $\varphi$, a descriptive $\mathrm{M}^{+} \mathrm{IPC}$-frame $\mathfrak{F}=(W, R, E)$, and a valuation $\nu$ on $\mathfrak{F}$ such that $\mathfrak{F} \not \models \varphi$. By modifying the construction in [20, $\S 10.3]$, we will construct a sequence of finite $\mathrm{M}^{+}$IPC-frames $\mathfrak{F}_{h}=\left(W_{h}, R_{h}, E_{h}\right)$ such that $\mathfrak{F}_{h} \subseteq \mathfrak{F}_{h+1}$ for all $h<\omega$. For each point $t \in W_{h}$ that we select, we will be creating a copy of some original point in $W$. We give each added point a new name, say $t$, and let $\widehat{t}$ denote the original point in $W$ that $t$ was copied from and will behave similar to. Thus, it is possible to have two different points $x_{1}$ and $x_{2}$ in our new frame, where $\widehat{x_{1}}=\widehat{x_{2}}$. The main difference 
between our construction and the construction given in [20, §10.3] will be seen in the $\rightarrow$-step, which requires a more careful selection of new points.

To start the construction, let $\mathfrak{F}_{0}=\left(W_{0}, R_{0}, E_{0}\right)$ where

$$
W_{0}=\left\{t_{0}\right\}, \quad R_{0}=W_{0}^{2}, \quad E_{0}=W_{0}^{2},
$$

and $\widehat{t_{0}}$ is a point in $W$ such that $\widehat{t_{0}}$ is from a clean cluster and is maximal with respect to $\varphi$. The existence of such $\widehat{t_{0}}$ follows from Lemma $5.2(4)$. Moreover, let $W_{-1}^{\forall H}=\varnothing$.

Let $\operatorname{Sub}(\varphi)$ be the set of subformulas of $\varphi$, and let $\left(W^{\prime}, R^{\prime}, E^{\prime}\right)$ be any of our frames in the construction. To each $t \in W^{\prime}$ we associate the following subsets of $\operatorname{Sub}(\varphi)$ :

$$
\begin{aligned}
\Sigma^{\exists}(t) & =\{\exists \delta \in \operatorname{Sub}(\varphi): \widehat{t} \vDash \exists \delta\} \\
\Sigma^{\forall H}(t) & =\{\forall \beta \in \operatorname{Sub}(\varphi): \widehat{t} \text { is maximal wrt } \forall \beta\} \\
\Sigma^{\forall V}(t) & =\{\forall \gamma \in \operatorname{Sub}(\varphi): \widehat{t} \not \forall \forall \gamma \text { but is not maximal wrt } \forall \gamma\} \\
\Sigma^{\rightarrow}(t) & =\{\alpha \rightarrow \sigma \in \operatorname{Sub}(\varphi): \widehat{t} \not \forall \alpha \rightarrow \sigma \text { but is not maximal wrt } \alpha \rightarrow \sigma\} .
\end{aligned}
$$

These are precisely the subformulas of $\varphi$ whose truth-value at $\widehat{t}$ is relevant for constructing our countermodel.

Suppose $\mathfrak{F}_{h-1}=\left(W_{h-1}, R_{h-1}, E_{h-1}\right)$ has already been constructed so that $\mathfrak{F}_{h-1}$ is a finite $\mathrm{M}^{+}$IPC-frame and $E(\widehat{w})$ is a clean cluster for each $w \in W_{h-1}$. We construct $\mathfrak{F}_{h}$ applying the four steps described below. They are designed to add the necessary witnesses required by the formulas in the sets $\Sigma^{\exists}(t), \Sigma^{\forall H}(t), \Sigma^{\forall V}(t)$, and $\Sigma^{\rightarrow}(t)$, respectively. In the $\exists$-step we ensure that for each formula in $\Sigma^{\exists}(t)$ the point $t$ has an $E$-successor that witnesses the existential statement. In the $\forall H$-step we ensure that for each formula in $\Sigma^{\forall H}(t)$ the point $t$ has an $E$-successor that witnesses the refutation of the universal statement. In the vertical steps $\forall V$ and $\rightarrow$ we make sure that $t$ has the necessary $R$-successors that are maximal with respect to the formulas in $\Sigma^{\forall V}(t)$ and $\Sigma^{\rightarrow}(t)$, respectively. In each step of the construction we add also points to witness commutativity. Note that the first three of the following four steps are only done once per cluster. This is enough since all points of a cluster in $\mathfrak{F}$ agree on refuting an $\forall$ - or $\exists$-formula and points from a clean cluster agree whether such a refutation is maximal.

Roughly speaking, points are added to the construction in the following order: In the first round the cluster of the starting point $t_{0}$ is built by adding points for formulas in $\Sigma^{\exists}\left(t_{0}\right)$ and $\Sigma^{\forall H}\left(t_{0}\right)$. After this, no more points are added to this cluster. We call this the 'bottom cluster' of our frame. The first round of the construction proceeds by adding vertical witnesses for each formula in $\Sigma^{\forall V}\left(t_{0}\right)$ and closing each such cluster by adding points for commutativity. The first round then finishes by adding for each point $t$ in the 'bottom cluster' vertical witnesses for the formulas in $\Sigma \rightarrow(t)$ and closing under commutativity. In the next round all these newly build clusters will possibly be enlarged in the horizontal steps and then new vertical clusters will be added in the $\forall V$ - and $\rightarrow$-steps.

$\exists$-step (Horizontal): Let $W_{h}^{\exists}=W_{h-1}, R_{h}^{\exists}=R_{h-1}$, and $E_{h}^{\exists}=E_{h-1}$. For each $E_{h}^{\exists}(t) \subseteq W_{h}^{\exists} \backslash W_{h-1}^{\forall H}$, if $\exists \delta \in \Sigma^{\exists}(t)$ but there is no $s \in W_{h}^{\exists}$ already such that $t E_{h}^{\exists} s$ and $\widehat{s} \vDash \delta$, then we add a point $s$ to $W_{h}^{\exists}$ with $\widehat{s} \models \delta$ and $\widehat{t} E \widehat{s}$. Such a point $\widehat{s}$ exists in $W$ since $\widehat{t}=\exists \delta$. We then add the ordered pairs $(s, s)$ to $R_{h}^{\exists}$, the ordered pairs $(t, s)$ to $E_{h}^{\exists}$, and generate the least equivalence relation.

$\forall H$-step (Horizontal): Let $W_{h}^{\forall H}=W_{h}^{\exists}, R_{h}^{\forall H}=R_{h}^{\exists}$, and $E_{h}^{\forall H}=E_{h}^{\exists}$. For each $E_{h}^{\forall H}(t) \subseteq W_{h}^{\forall H} \backslash W_{h-1}^{\forall H}$, if $\forall \beta \in \Sigma^{\forall H}(t)$ but there is no $s \in W_{h}^{\forall H}$ already such that $t E_{h}^{\forall H} s$ and $\widehat{s} \not \beta \beta$, then we add a point $s$ to $W_{h}^{\forall H}$ with $\widehat{s} \not \forall \beta$ and $\widehat{t} E \widehat{s}$. Such a point $\widehat{s}$ exists in $W$ since $\widehat{t}$ is maximal with respect to $\forall \beta$. We then add the ordered pairs $(s, s)$ to $R_{h}^{\forall H}$, the ordered pairs $(t, s)$ to $E_{h}^{\forall H}$, and generate the least equivalence relation.

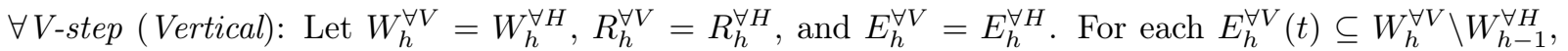
consider $\forall \gamma \in \Sigma^{\forall V}(t)$. Since $\widehat{t} \not \forall \forall \gamma$, we can pick a point $\widehat{s} \in W$ as in Lemma 5.2(2). We add the point $s$ to $W_{h}^{\forall V}$ and $(t, s)$ to $R_{h}^{\forall V}$.

Since $W$ satisfies commutativity, for each $w \in E_{h}^{\forall V}(t)$, there is $z_{w} \in W$ such that $\widehat{w} R z_{w}$ and $z_{w} E \widehat{s}$. To ensure commutativity is satisfied in our new frame, we add the points $s_{w}$ to $W_{h}^{\forall V}$ where $\widehat{s_{w}}=z_{w}$. We then add $\left(w, s_{w}\right)$ to $R_{h}^{\forall V}$ and take the reflexive and transitive closure. We also add $\left(s_{w}, s\right)$ to $E_{h}^{\forall V}$ and generate the least equivalence relation. 
$\rightarrow$-step (Vertical): Let $W_{h} \rightarrow=W_{h}^{\forall V}, R_{h}^{\rightarrow}=R_{h}^{\forall V}$, and $E_{h} \rightarrow=E_{h}^{\forall V}$. For each $t \in W_{h}^{\forall H} \backslash W_{h-1}^{\forall H}$ (hence including any points added in the horizontal steps above, but not in the previous vertical step), consider all $\alpha \rightarrow \sigma \in \Sigma^{\rightarrow}(t)$ such that there is no $s \in W_{h} \rightarrow$ already such that $t R_{h} s$ and $\widehat{s} \not \forall \alpha \rightarrow \sigma$ maximally. Consider

$$
A=[W \backslash \nu(\alpha \rightarrow \sigma)] \cap \bigcap_{\psi \in \operatorname{Sub}(\varphi)}\{\nu(\psi): \widehat{t} \vDash \psi\} .
$$

Then $A$ is clopen and $\widehat{t} \in A$, so by Lemma $5.2(3)$ there is $z \in A$ with $z \in \max E(A), \widehat{t} Q z$, and $E(z)$ clean. We add the point $s$ to $W_{h}$ where $\widehat{s}=z(s$ is a distinct new copy of $z)$ and $(t, s)$ to $R_{h}$.

Remark 5.3. It is at this step that we have altered the construction given in [20, §10.3], in which witnesses for implications are added in the same manner as in the $\forall V$-step. In our version, we took an original $Q$ relation and turned it into an $R$-relation. The reason for this is that we cannot guarantee the existence of an $R$-successor of $t$ that is maximal with respect to $\alpha \rightarrow \sigma$ and at the same time belongs to a clean cluster.

Before wrapping up the step, we show two properties of the chosen points.

Lemma 5.4. The point $\widehat{s}=z$, as chosen above, is maximal with respect to $\alpha \rightarrow \sigma$.

Proof. Suppose $z R u$ for some $u \not \models \alpha \rightarrow \sigma$. Since $z R u$ and each $\nu(\psi)$ in $\{\nu(\psi): \widehat{t} \vDash \psi\}$ is an upset, we have $u \in A$. Because $z \in \max A$, we obtain $z=u$. Thus, $z$ is maximal with respect to $\alpha \rightarrow \sigma$.

Lemma 5.5. $E(\widehat{t}) \neq E(\widehat{s})$.

Proof. If $E(\widehat{t})=E(\widehat{s})$, then $\widehat{t} \in \max E(A)$ by Lemma 5.1. Since $\widehat{t} \in A$, we have $\widehat{t} \in \max A$. Therefore, the same argument as in the proof of the previous lemma yields that $\widehat{t}$ is maximal with respect to $\alpha \rightarrow \sigma$. This contradicts $\alpha \rightarrow \sigma \in \Sigma \rightarrow(t)$.

We wrap up the $\rightarrow$-step the same way as the $\forall V$-step. Since $W$ satisfies commutativity, for each $w \in$ $E_{h}^{\forall V}(t)$ there is $z_{w} \in W$ with $\widehat{w} R z_{w}$ and $z_{w} E \widehat{s}$. We add the points $s_{w}$ to $W_{h}^{\forall V}$ where $\widehat{s_{w}}=z_{w}$. We then add $\left(w, s_{w}\right)$ to $R_{h}$ and take the reflexive and transitive closure. We also add $\left(s_{w}, s\right)$ to $E_{h} \rightarrow$ and generate the least equivalence relation.

To end this stage of the construction, we let $\mathfrak{F}_{h}=\left(W_{h}, R_{h}, E_{h}\right)$ where

$$
W_{h}=W_{h}, \quad R_{h}=R_{h}, \quad E_{h}=E_{h} \rightarrow
$$

Lemma 5.6. $\mathfrak{F}_{h}$ is a finite $\mathrm{M}^{+} \mathrm{IPC}$-frame.

Proof. First we show that $R_{h}$ is a partial order. Since in the $\exists$ - and $\forall H$-steps we only added reflexive arrows to $R_{h-1}$, the relation $R_{h}^{\forall H}$ is a partial order. By moving from $R_{h}^{\forall H}$ to $R_{h}$ we finished by taking the reflexive and transitive closure, hence $R_{h}$ is clearly reflexive and transitive. Antisymmetry of $R_{h}$ follows from the fact that every $R$-arrow added in the $\forall V$-step and $\rightarrow$-step is either reflexive or an arrow from a previously existing point into a freshly added point.

That $E_{h}$ is an equivalence relation is clear from the construction. Moreover, the extra points added in the $\forall V$-step and $\rightarrow$-step make sure that commutativity is satisfied. In fact, the added points assure commutativity for immediate successors and by transitivity this implies commutativity for the whole frame. Therefore, $\mathfrak{F}_{h}$ is an MIPC-frame.

It follows from the construction that $\mathfrak{F}_{h}$ is finite. Thus, by Lemma 4.4 , it is left to show that $\mathfrak{F}_{h}$ has clean clusters. Note that in the $\exists$-step and $\forall H$-step all freshly introduced $E_{h}$-relations are of the shape $(s, t)$ where either $s \in W_{h}$ and $t \in W_{h}^{\forall H} \backslash W_{h-1}$ or $s, t \in W_{h}^{\forall H}$. Since no non-reflexive $R_{h}$-arrows are introduced in these steps, no dirty cluster could have been built. We have already discussed the shape of the $R_{h}$ arrows introduced in the $\forall V$-step and $\rightarrow$-step. This guarantees that no cluster in $W_{h}^{\forall H}$ is made dirty. The freshly introduced $E_{h}$-relations in these steps are of the shape $(s, t)$ where $s, t \in W_{h} \backslash W_{h}^{\forall H}$. Since no non-reflexive $R_{h}$ relations exist between these points, we infer that all clusters are clean.

5.2. Auxiliary lemmas. To prove that our construction terminates after finitely many steps, we require several auxiliary lemmas.

Lemma 5.7. Let $x, y \in W_{h}$.

(1) If $x R_{h} y$ and $x \neq y$, then $\widehat{x} Q \widehat{y}$ and $E(\widehat{x}) \neq E(\widehat{y})$.

(2) If $x E_{h} y$, then $\widehat{x} E \widehat{y}$. 


\section{(3) If $x Q_{h} y$, then $\widehat{x} Q \widehat{y}$.}

Proof. (1) Observe that in the construction each non-trivial $R_{h}$-relation between immediate successors comes from either a non-trivial $R$-relation (as in the case of points added for commutativity or in the $\forall V$-step) or a non-trivial $Q$-relation (as in the case of points added in the $\rightarrow$-step), in which case there is $w \in W$ with $\widehat{x} \neq w, \widehat{x} R w$, and $w E \widehat{y}$. In that case we obviously have $\widehat{x} Q \widehat{y}$ and by Lemma $5.5, E(\widehat{x}) \neq E(\widehat{y})$ in $W$. Otherwise the relation $x R_{h} y$ was added by transitivity, so there is a chain $x=x_{0} R_{h} x_{1} R_{h} \ldots R_{h} x_{n}=y$ of immediate $R_{h}$-successors to which the previous applies. In particular, by transitivity of $Q$ we have $\widehat{x_{i}} Q \widehat{y}$ for all $i \leq n$, so $\widehat{x} Q \widehat{y}$. Moreover, there is $z_{1} \in W$ with $z_{1} \neq x, \widehat{x} R z_{1}$ and $z_{1} E \widehat{x_{1}}$. Since $\widehat{x_{1}} Q \widehat{y}$, by commutativity there is $y^{\prime}$ in $W$ with $z_{1} R y^{\prime}$ and $y^{\prime} E \widehat{y}$. If $\widehat{x} E \widehat{y}$, then also $\widehat{x} E y^{\prime}$ and $\widehat{x} R y^{\prime}$ by transitivity of $R$. Since $\widehat{x}$ is from a clean cluster, this implies $\widehat{x}=y^{\prime}$. Therefore, $\widehat{x} R z_{1} R \widehat{x}$, and so $\widehat{x}=z_{1}$ by antisymmetry of $R$. This is a contradiction since $\widehat{x} \neq z_{1}$. Thus, $E(\widehat{x}) \neq E(\widehat{y})$.

(2) It is obvious that each $E_{h}$-relation in $W_{h}$ comes from a pre-existing $E$-relation in $W$.

(3) If $x Q_{h} y$, then there is $z$ with $x R_{h} z$ and $z E_{h} y$. If $x=z$, then $x E_{h} y$, so $\widehat{x} E \widehat{y}$ by (2), and hence $\widehat{x} Q \widehat{y}$. If $x \neq z$, then $\widehat{x} Q \widehat{z}$ by (1). Also, $z E_{h} y$ implies $\widehat{z} E \widehat{y}$ by (2). Thus, $\widehat{x} Q \widehat{y}$.

Lemma 5.8 (Persistence). If $u R_{h} w$, then $\widehat{u} \vDash \psi$ implies $\widehat{w} \vDash \psi$ for all $\psi \in \operatorname{Sub}(\varphi)$.

Proof. Suppose $u R_{h} w, \psi \in \operatorname{Sub}(\varphi)$, and $\widehat{u} \vDash \psi$. It suffices to show the result for an immediate $R_{h}$-successor $w$ of $u$, the general result then follows by induction. We consider how the $R_{h}$-arrow from $u$ to $w$ was added. By construction, either $\widehat{u} R \widehat{w}$ or $w$ was added to witness some implication in $\Sigma^{\rightarrow}(u)$. If $\widehat{u} R \widehat{w}$, then clearly $\widehat{u} \vDash \psi$ implies $\widehat{w} \vDash \psi$. If $w$ was added in a $\rightarrow$-step, then $w$ is specifically chosen so that $\widehat{w} \in \nu(\gamma)$ for all $\gamma \in \operatorname{Sub}(\varphi)$ such that $\widehat{u} \vDash \gamma$. Thus, $\widehat{u} \vDash \psi$ implies $\widehat{w} \vDash \psi$.

\section{Lemma 5.9.}

(1) If $t E_{h} u$, then $\Sigma^{\exists}(t)=\Sigma^{\exists}(u), \Sigma^{\forall H}(t)=\Sigma^{\forall H}(u)$, and $\Sigma^{\forall V}(t)=\Sigma^{\forall V}(u)$.

(2) If $t R_{h} v$ and $\exists \gamma \in \Sigma^{\exists}(t) \cap \Sigma^{\exists}(v)$, then there are $u, w$ such that $t E_{h} u, u R_{h} w, w E_{h} v, \widehat{u} \models \gamma$, and $\widehat{w}=\gamma$.

(3) If $t R_{h} v$ and $t \neq v$, then $\Sigma^{\forall H}(t) \cap \Sigma^{\forall H}(v)=\varnothing$.

(4) Along an $R_{h}$-chain, each formula in $\{\forall \psi: \forall \psi \in \operatorname{Sub}(\varphi)\} \cup\{\exists \psi: \exists \psi \in \operatorname{Sub}(\varphi)\}$ can serve at most once as a reason to enlarge a cluster in a horizontal step.

(5) If $t R_{h} u$, then $\Sigma^{\forall V}(u) \subseteq \Sigma^{\forall V}(t)$ and if $u$ was added as an immediate $R_{h}$-successor to $t$ because of $\forall \alpha \in \Sigma^{\forall V}(t)$, then $\Sigma^{\forall V}(u) \subset \Sigma^{\forall V}(t)$.

(6) If $t R_{h} u$, then $\Sigma^{\rightarrow}(u) \subseteq \Sigma^{\rightarrow}(t)$ and if $u$ was added as an immediate $R_{h}$-successor to $t$ because of $\alpha \rightarrow \beta \in \Sigma^{\rightarrow}(t)$, then $\Sigma \rightarrow(u) \subset \Sigma^{\rightarrow}(t)$.

Proof. (1) Suppose $t E_{h} u$. Then $\widehat{t} E \widehat{u}$ by Lemma 5.7(2). Therefore, $E(\widehat{t})=E(\widehat{u})$ and $Q(\widehat{t})=Q(\widehat{u})$. Thus, $\widehat{t} \vDash \exists \gamma$ iff $\widehat{u} \vDash \exists \gamma$, and $\widehat{t} \vDash \forall \gamma$ iff $\widehat{u} \vDash \forall \gamma$. Moreover, since $E(\widehat{t})$ is a clean cluster, $\widehat{t}$ is not maximal wrt $\forall \gamma$ iff $\widehat{u}$ is not maximal wrt $\forall \gamma$. Consequently, $\Sigma^{\exists}(t)=\Sigma^{\exists}(u), \Sigma^{\forall H}(t)=\Sigma^{\forall H}(u)$, and $\Sigma^{\forall V}(t)=\Sigma^{\forall V}(u)$.

(2) Suppose $t R_{h} v$ and $\exists \gamma \in \Sigma^{\exists}(t) \cap \Sigma^{\exists}(v)$. By the construction, there is $u$ with $t E_{h} u$ and $\widehat{u} \vDash \gamma$. Since $\mathfrak{F}_{h}$ satisfies commutativity, there is $w$ with $u R_{h} w$ and $w E_{h} v$. By Lemma $5.8, \widehat{w} \vDash \gamma$.

(3) Suppose $t R_{h} v$ and $t \neq v$. Then $\widehat{t} Q \widehat{v}$ and $E(\widehat{t}) \neq E(\widehat{v})$ by Lemma 5.7 $(1)$, so $\widehat{t} \neq \widehat{v}$. Thus, if $\forall \psi \in \Sigma^{\forall H}(t)$, then $\widehat{v} \vDash \forall \psi$ by maximality of $\widehat{t}$, so $\forall \psi \notin \Sigma^{\forall H}(v)$. Conversely, if $\forall \psi \in \Sigma^{\forall H}(v)$, then $\widehat{t}$ cannot be maximal with respect to $\forall \psi$, so $\forall \psi \notin \Sigma^{\forall H}(t)$.

(4) Let $\left\{v_{i} \mid i \in \mathbb{N}\right\}$ be an $R_{h}$-chain in $W_{h}$, i.e. $v_{i} R_{h} v_{i+1}$ for all $i \in \mathbb{N}$. Suppose $\exists \psi \in \operatorname{Sub}(\varphi)$. Let $k$ be the least stage at which the formula $\exists \psi$ has been used to enlarge the cluster $E_{h}\left(v_{k}\right)$ in a horizontal step. By (2), all $E_{h}\left(v_{l}\right)$ for $l>k$ already contain a witness for $\psi$, so no cluster above will need to be enlarged in a horizontal step to witness the formula $\exists \psi$. Now suppose $\forall \psi \in \operatorname{Sub}(\varphi)$. Let $l$ be a stage at which the formula $\forall \psi$ has been used to enlarge the cluster $E_{h}\left(v_{l}\right)$ in a horizontal step. Then $\forall \psi \in \Sigma^{\forall H}\left(v_{l}\right)$. By (3), $\forall \psi \notin \Sigma^{\forall H}\left(v_{k}\right)$ for $k \neq l$. Thus, $\forall \psi$ is responsible for enlarging a cluster at most once in a horizontal step.

(5) We show the statement for immediate $R_{h}$-successors only, the general case follows by induction. Suppose $t R_{h} u$ and $\forall \psi \in \Sigma^{\forall V}(u)$. If $t=u$, then the result is clear. Suppose $t \neq u$. Since $t R_{h} u$, either $\widehat{t} R \widehat{u}$ in $W$ or $u$ was added as a successor of $t$ in some $\rightarrow$-step. If $\widehat{t} R \widehat{u}$, then $\forall \psi \in \Sigma^{\forall V}(t)$ by persistence (see Lemma 5.8). Suppose $u$ was added as an $R_{h}$-successor to $t$ as a witness to some implication. By the choice of $u$, we have $\widehat{u} \vDash \chi$ for all $\chi \in \operatorname{Sub}(\varphi)$ with $\widehat{t} \vDash \chi$. Therefore, if $\widehat{t} \vDash \forall \psi$, then we would have $\widehat{u} \vDash \forall \psi$, contradicting $\forall \psi \in \Sigma^{\forall V}(u)$. Thus, we must have $\widehat{t} \not \forall \forall \psi$. Moreover, since $\widehat{t} R \widehat{u}, \widehat{t} \neq \widehat{u}$, and $\widehat{u} \not \forall \forall \psi$, we 
have that $\widehat{t}$ is not maximal with respect to $\forall \psi$, so $\forall \psi \in \Sigma^{\forall V}(t)$. Consequently, in either case we have $\Sigma^{\forall V}(u) \subseteq \Sigma^{\forall V}(t)$.

Suppose that $u$ was added as an immediate $R_{h}$-successor to $t$ because of $\forall \alpha \in \Sigma^{\forall V}(t)$. Since $\forall \alpha \in \Sigma^{\forall V}(t)$, we have $\widehat{t} \not \forall \forall \alpha$ but $\widehat{t}$ is not maximal with respect to $\forall \alpha$. Since $u$ was added as an immediate $R_{h}$-successor of $t$ because of $\forall \alpha$, we specifically chose $u$ so that $\widehat{u} \not \forall \forall \alpha$ maximally, hence $\forall \alpha \notin \Sigma^{\forall V}(u)$.

(6) We show the statement for immediate $R_{h}$-successors only, the general case follows by induction. Suppose $t R_{h} u$ and $\alpha \rightarrow \beta \in \Sigma \rightarrow(u)$. Then $\widehat{u} \not \forall \alpha \rightarrow \beta$ and $\widehat{u} \not \forall \alpha$. If $t=u$, then the result is clear. Suppose $t \neq u$. Since $t R_{h} u$, either $\widehat{t} R \widehat{u}$ in $W$ or $u$ was added as a successor of $t$ in some $\rightarrow$-step. If $\widehat{t} R \widehat{u}$, then $\alpha \rightarrow \beta \in \Sigma \rightarrow(t)$ by persistence (see Lemma 5.8). Suppose $u$ was added as an $R_{h}$-successor to $t$ as a witness to some implication. By the choice of $u$, we have $\widehat{u} \vDash \psi$ for all $\psi \in \operatorname{Sub}(\varphi)$ with $\widehat{t} \vDash \psi$. Therefore, we must have $\widehat{t} \not=\alpha \rightarrow \beta$ and $\widehat{t} \not \forall \alpha$, so $\Sigma \rightarrow(u) \subseteq \Sigma \rightarrow(t)$. Moreover, by construction, $\widehat{u}$ refutes $\alpha \rightarrow \beta$ maximally (Lemma 5.4), and hence $\widehat{u} \vDash \alpha$. Thus, $\alpha \rightarrow \beta \notin \Sigma \rightarrow(u)$.

5.3. Termination of the construction. With the aid of the auxiliary lemmas of the previous section, we will now prove that the end result of our construction is a finite frame. We will do this by looking at three important parameters of our frame: cluster size, $R$-branching, and $R$-depth.

\section{Definition 5.10.}

(1) A frame $\mathfrak{F}$ has bounded cluster size if there exists $k \in \mathbb{N}$ such that $|E(t)| \leq k$ for all $t \in W$.

(2) A frame $\mathfrak{F}$ has bounded R-branching if there exists $m \in \mathbb{N}$ such that $t$ has at most $m$ distinct immediate $R$-successors for all $t \in W$.

(3) A frame $\mathfrak{F}$ has bounded $R$-depth if there exists $n \in \mathbb{N}$ such that there is no $R$-chain in $\mathfrak{F}$ with more than $n$ distinct elements.

Lemma 5.11. Let $\mathfrak{F}=(W, R, E)$ be a partially ordered rooted augmented Kripke frame. If $\mathfrak{F}$ has bounded cluster size, bounded $R$-branching, and bounded $R$-depth, then $\mathfrak{F}$ is finite.

Proof. Suppose $\mathfrak{F}=(W, R, E)$ is a partially ordered rooted augmented Kripke frame with bounded cluster size, $R$-branching, and $R$-depth. Consider the quotient $\left(W / E, R_{E}\right)$ whose worlds are the clusters $E(x)$ where $x \in W$ and $E(x) R_{E} E(y)$ iff $x Q y$. To see that $R_{E}$ is well defined, suppose $x Q y, x^{\prime} \in E(x)$, and $y^{\prime} \in E(y)$. Then $x^{\prime} E x Q y E y^{\prime}$, so $x^{\prime} Q y^{\prime}$, and hence $R_{E}$ is well defined.

Because $Q$ is reflexive and transitive, so is $R_{E}$. Since $R$ is a partial order and $\mathfrak{F}$ has bounded $R$-depth, from $x Q y$ and $y Q x$ it follows that $x E y$ by [3, Lem. 3(b)]. This shows that $R_{E}$ is anti-symmetric, and hence a partial order. Clearly $\left(W / E, R_{E}\right)$ is rooted since so is $\mathfrak{F}$. Using commutativity in $\mathfrak{F}$ it is easy to verify that $\left(W / E, R_{E}\right)$ inherits bounded depth and bounded branching from $\mathfrak{F}$. Since every rooted partial order with the latter properties is finite, we have that $W / E$ is finite. Because $W$ has bounded cluster size, we conclude that $W$ is finite too.

Let $m_{1}, m_{2}, m_{3}$ be the non-negative integers

$$
\begin{aligned}
& m_{1}=|\{\exists \psi: \exists \psi \in \operatorname{Sub}(\varphi)\}| \\
& m_{2}=|\{\forall \psi: \forall \psi \in \operatorname{Sub}(\varphi)\}| \\
& m_{3}=|\{\psi \rightarrow \chi: \psi \rightarrow \chi \in \operatorname{Sub}(\varphi)\}| .
\end{aligned}
$$

Lemma 5.12. $\mathfrak{F}_{h}=\left(W_{h}, R_{h}, E_{h}\right)$ has cluster size bounded by $1+m_{1}+m_{2}$ for all $h<\omega$.

Proof. Recall how the clusters of our frame are built. The 'bottom cluster' of the starting point $t_{0}$ contains points added via the horizontal $\exists$ - and $\forall H$-steps. After this, no more points are added to this cluster.

All other clusters are constructed as follows. First points of a new cluster are added via the vertical $\forall V$ or $\rightarrow$-steps, and then the cluster is enlarged by the points added for commutativity. We refer to this stage as the 'building phase' of the cluster. In the next round of the construction, the cluster is (possibly) enlarged via the two horizontal steps. After this, no more points are added to the cluster. In the horizontal steps, we enlarge the cluster for only two different reasons:

$$
\exists \gamma \in \Sigma^{\exists}(t) \text { or } \forall \gamma \in \Sigma^{\forall H}(t) .
$$

Thus, each enlargement of a cluster after its building phase is due to a formula in

$$
\{\forall \psi: \forall \psi \in \operatorname{Sub}(\varphi)\} \cup\{\exists \psi: \exists \psi \in \operatorname{Sub}(\varphi)\} .
$$


At the end of its building phase, the bottom cluster contains just one point. Observe that every cluster can be reached from the bottom cluster by an $R_{h}$-chain. By Lemma 5.9(4), every formula in $\{\forall \psi: \forall \psi \in$ $\operatorname{Sub}(\varphi)\} \cup\{\exists \psi: \exists \psi \in \operatorname{Sub}(\varphi)\}$ can serve at most once as a reason to enlarge a cluster after its building phase along an $R_{h}$-chain. This entails that every cluster has size at most $1+m_{1}+m_{2}$.

Lemma 5.13. $\mathfrak{F}_{h}=\left(W_{h}, R_{h}, E_{h}\right)$ has $R_{h}$-branching bounded by $\left(1+m_{1}+m_{2}\right) \cdot m_{3}+m_{2}$ for all $h<\omega$.

Proof. Immediate $R_{h}$-successors are added in the $\forall V$-step and $\rightarrow$-step. First observe that since we are adding points to witness commutativity, every point in a cluster has the same number of immediate $R_{h}$-successors by the end of a stage. Thus, it is enough to count the immediate successors of a point $t$ that we picked in the $\forall V$-step.

To such a point $t$ we add immediate $R_{h}$-successors for three different reasons:

(1) $\forall \gamma \in \Sigma^{\forall V}(t)$,

(2) $\alpha \rightarrow \sigma \in \Sigma \rightarrow(t)$, or

(3) $\alpha \rightarrow \sigma \in \Sigma^{\rightarrow}(y)$ for some $y \in E_{h}(t)$ with $y \neq t$.

The last reason covers the case where we add an $R_{h}$-successor to $t$ to witness commutativity. Note that all reasons occur at most once for each formula in the respective sets. Therefore, reason (1) occurs at most $m_{2}$-times and reason (2) at most $m_{3}$-times. Finally, reason (3) occurs at most $\left(m_{1}+m_{2}\right) \cdot m_{3}$ times since by Lemma 5.12 there are at most $m_{1}+m_{2}$ points apart from $t$ in the cluster of $t$. Thus, the $R_{h}$-branching of $\mathfrak{F}$ is bounded by

$$
m_{2}+m_{3}+\left(m_{1}+m_{2}\right) \cdot m_{3}=m_{2}+\left(1+m_{1}+m_{2}\right) \cdot m_{3}
$$

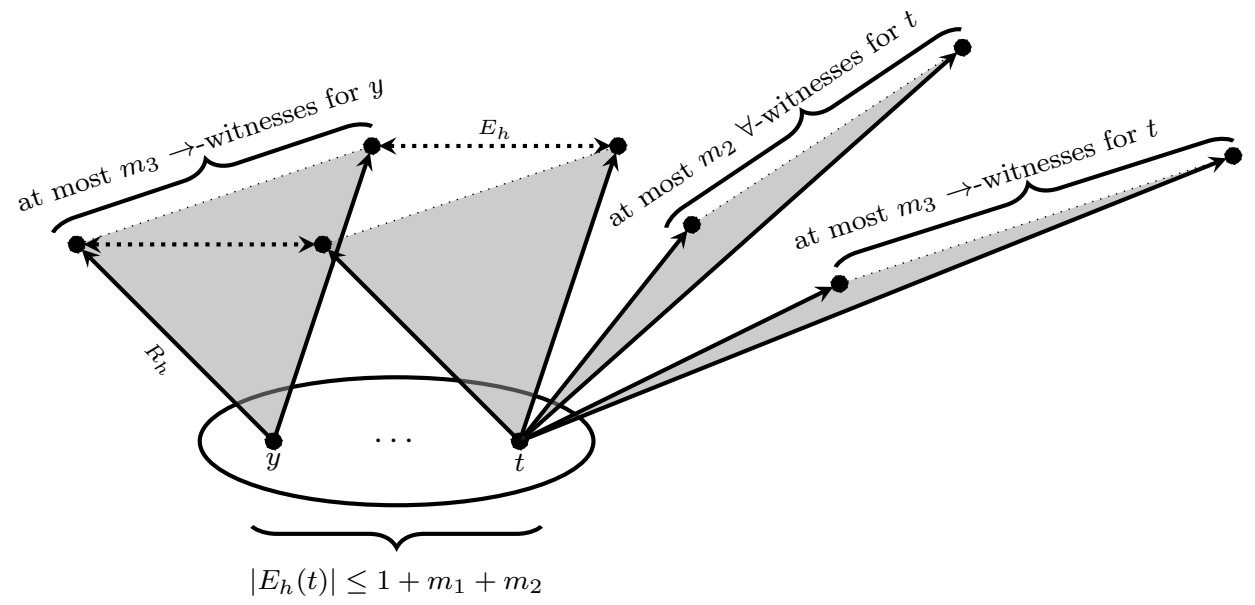

Lemma 5.14. $\mathfrak{F}_{h}=\left(W_{h}, R_{h}, E_{h}\right)$ has $R_{h}$-depth bounded by $\left(1+m_{1}+m_{2}\right) \cdot\left(m_{2}+m_{3}\right)$ for all $h<\omega$.

Proof. The reason for adding an immediate successor to $t \in W_{h}$ via an $R_{h}$-relation is due to either a formula in $\Sigma^{\forall V}(t)$ or a formula in $\Sigma \rightarrow(y)$ for some $y \in E_{h}(t)$ (as discussed in the proof of Lemma 5.13). Let $s$ be a (not necessarily immediate) $R_{h}$-successor of $t$. Then $s$ could have been added via direct formula witnessing, i.e. there is an immediate predecessor $t^{\prime}$ of $s$ with $t R_{h} t^{\prime} R_{h} s$ and $s$ was added due to a formula in $\Sigma^{\forall V}\left(t^{\prime}\right)$ or $\Sigma \rightarrow\left(t^{\prime}\right)$, or else $s$ was added to satisfy commutativity.

As we saw in Lemma 5.9 moving up along an $R_{h}$-chain, the cardinality of the sets $\Sigma^{\forall V}(t)$ and $\Sigma \rightarrow(t)$ does not increase, and it in fact decreases whenever an $R_{h}$-successor is added by direct formula witnessing. In particular, each point can have at most $m_{2}+m_{3} R_{h}$-successors that have been added via direct formula witnessing and since in each cluster there are at most $1+m_{1}+m_{2}$ points (Lemma 5.12 , we have that the total $R_{h}$-depth cannot exceed $\left(1+m_{1}+m_{2}\right) \cdot\left(m_{2}+m_{3}\right)$. 


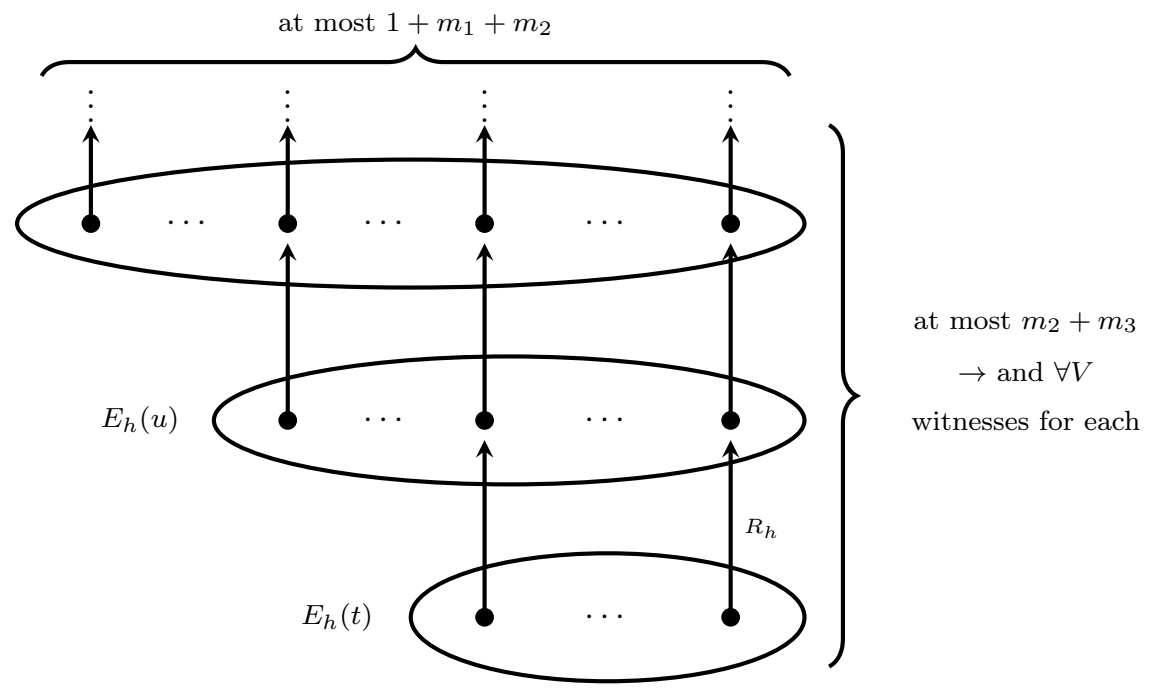

Lemma 5.15. There is $h \in \mathbb{N}$ such that $\mathfrak{F}_{h^{\prime}}=\mathfrak{F}_{h}$ for all $h^{\prime} \geq h$.

Proof. All points in the bottom cluster are added in round 1 and in each round we enlarge the $R_{h}$-length of a path by at most one. Thus, in stage $k$ of the construction, all $R_{h}$-chains are bounded by $k$. The construction continues only until vertical witnesses are required. Since, by Lemma 5.14, the $R_{h}$-depth of $\mathfrak{F}_{k}$ is bounded by $m=\left(1+m_{1}+m_{2}\right) \cdot\left(m_{2}+m_{3}\right)$, we have $\mathfrak{F}_{h^{\prime}}=\mathfrak{F}_{m+1}$ for all $h^{\prime} \geq m+1$.

Set $\mathfrak{F}^{\prime}=\left(W^{\prime}, R^{\prime}, E^{\prime}\right)$ where

$$
W^{\prime}=W_{h}, \quad R^{\prime}=h, \quad E^{\prime}=E_{h},
$$

and $h$ is as in Lemma 5.15. Then $\mathfrak{F}^{\prime}$ is a finite $\mathrm{M}^{+}$IPC-frame by Lemma 5.6.

5.4. Truth lemma. Define a valuation $\nu^{\prime}$ on $W^{\prime}$ by $\nu^{\prime}(p)=\left\{t \in W^{\prime}: \widehat{t} \in \nu(p)\right\}$ for $p \in \operatorname{Sub}(\varphi)$ and $\nu^{\prime}(q)=\varnothing$ for variables $q$ not occurring in $\varphi$. That $\nu^{\prime}$ is well defined follows from Lemma 5.8, which ensures that the sets $\nu^{\prime}(\psi)$ are in $\operatorname{Up}\left(\mathfrak{F}^{\prime}\right)$ for each $\psi \in \operatorname{Sub}(\varphi)$.

Lemma 5.16 (Truth Lemma). For all $t \in W^{\prime}$ and $\psi \in \operatorname{Sub}(\varphi)$, we have $t \vDash^{\prime} \psi$ iff $\widehat{t} \vDash \psi$.

Proof. The proof is by induction on the complexity of $\psi$. The base cases $\psi=\perp$ and $\psi=p$ ( $p$ a propositional variable) follow from the definition, and the cases $\psi=\psi_{1} \wedge \psi_{2}$ and $\psi=\psi_{1} \vee \psi_{2}$ are easily verified. So we focus on the cases $\psi=\psi_{1} \rightarrow \psi_{2}$ (and hence $\psi=\neg \psi_{1}=\psi_{1} \rightarrow \perp$ ), $\psi=\exists \psi_{1}$, and $\psi=\forall \psi_{1}$.

$\rightarrow$ case: Let $\psi=\psi_{1} \rightarrow \psi_{2}$ and $t \in W^{\prime}$. Suppose $t \not \not^{\prime} \psi_{1} \rightarrow \psi_{2}$. Then $t R^{\prime} s$ for some $s \in W^{\prime}$ with $s \vDash^{\prime} \psi_{1}$ $\overrightarrow{\text { and } s \not \not^{\prime}} \psi_{2}$. By the inductive hypothesis, $\widehat{s} \vDash \psi_{1}$ and $\widehat{s} \not \models \psi_{2}$. Thus, $\widehat{s} \not \models \psi_{1} \rightarrow \psi_{2}$. Since $t R^{\prime} s$, we have $\widehat{t} \not \forall \psi_{1} \rightarrow \psi_{2}$ by persistence (Lemma 5.8).

Conversely, suppose $\widehat{t} \not \forall \psi_{1} \rightarrow \psi_{2}$. If $\widehat{t} \vDash \psi_{1}$, then we have $\widehat{t} \vDash \psi_{1}$ but $\widehat{t} \not \psi_{2}$. By the inductive hypothesis, $t \vDash^{\prime} \psi_{1}$ but $t \not \not^{\prime} \psi_{2}$. By construction, $t R^{\prime} t$, so $t \not \not^{\prime} \psi_{1} \rightarrow \psi_{2}$. If $\widehat{t} \not \not \psi_{1}$, then in the $\rightarrow$-step of the stage immediately after $t$ is added to $W^{\prime}$, we add $s$ to $W^{\prime}$ and $t R^{\prime} s$ where $\widehat{s} \not \forall \psi_{1} \rightarrow \psi_{2}$ maximally (Lemma 5.4). Thus, $\widehat{s} \vDash \psi_{1}$ and $\widehat{s} \not \psi_{2}$, so by the inductive hypothesis, $s \vDash^{\prime} \psi_{1}$ and $s \not \not^{\prime} \psi_{2}$. Since $t R^{\prime} s$, we conclude that $t \not \not^{\prime} \psi_{1} \rightarrow \psi_{2}$.

$\exists$ case: Let $\psi=\exists \psi_{1}$ and $t \in W^{\prime}$. Suppose $t \vDash^{\prime} \exists \psi_{1}$. Then $t E^{\prime} s$ for some $s \in W^{\prime}$ with $s \vDash^{\prime} \psi_{1}$. By the inductive hypothesis, $\widehat{s} \vDash \psi_{1}$, and $t E^{\prime} s$ implies $\widehat{t} E \widehat{s}$ by Lemma $5.7(2)$. Thus, $\widehat{t} \vDash \exists \psi_{1}$.

Conversely, suppose $\widehat{t} \vDash \exists \psi_{1}$. Then $\exists \psi_{1} \in \Sigma^{\exists}(t)$, so in the $\exists$-step of the next stage of the construction after $t$ is added, we add $s$ to $W^{\prime}$ and $(t, s)$ to $E^{\prime}$ where $s$ is a copy of some $\widehat{s} \in W$ with $\widehat{t} E \widehat{s}$ and $\widehat{s} \vDash \psi_{1}$. By the inductive hypothesis, $s \models^{\prime} \psi_{1}$. Since $t E^{\prime} s$, we conclude that $t \models^{\prime} \exists \psi_{1}$.

$\forall$ case: Let $\psi=\forall \psi_{1}$ and $t \in W^{\prime}$. Suppose $t \not \not^{\prime} \forall \psi_{1}$. Then $t Q^{\prime} w$ for some $w \in W^{\prime}$ with $w \not \not^{\prime} \psi_{1}$. By the inductive hypothesis, $\widehat{w} \not \forall \psi_{1}$, and $t Q^{\prime} w$ implies $\widehat{t} Q \widehat{w}$ by Lemma 5.7(3). Thus, $\widehat{t} \not \forall \forall \psi_{1}$. 
Conversely, suppose $\widehat{t} \not \forall \forall \psi_{1}$. If $\widehat{t}$ is maximal with respect to $\forall \psi_{1}$, then $\forall \psi_{1} \in \Sigma^{\forall H}(t)$, so at some point in the construction of the next stage after $t$ is added, we add $s$ to $W^{\prime}$ and $(t, s)$ to $E^{\prime}$ where $s$ is a copy of some $\widehat{s} \in W$ with $\widehat{t} E \widehat{s}$ and $\widehat{s} \not \forall \psi_{1}$. By the inductive hypothesis, $s \not \not^{\prime} \psi_{1}$, so $t \not^{\prime} \psi_{1}$. If $\widehat{t}$ is not maximal, then we add $s$ to $W^{\prime}$ and $(t, s)$ to $R^{\prime}$ where $s$ is a copy of some $\widehat{s} \in W$ and $\widehat{s}$ is maximal with respect to $\forall \psi_{1}$. Therefore, $\forall \psi_{1} \in \Sigma^{\forall H}(s)$, and in the next stage we add $w$ to $W^{\prime}$ and $(s, w)$ to $E^{\prime}$ where $\widehat{w} \in W$ and $\widehat{w} \not \forall \psi_{1}$. But then $t Q^{\prime} w$, hence $\widehat{t} Q \widehat{w}$ (see Lemma 5.7), and by the inductive hypothesis, $w \not^{\prime} \psi_{1}$. Thus, $t \not^{\prime} \forall \psi_{1}$.

The FMP of $\mathrm{M}^{+} \mathrm{IPC}$ is now an immediate consequence of the above.

Theorem 5.17. $\mathrm{M}^{+} \mathrm{IPC}$ has the finite model property.

Proof. Suppose $\mathrm{M}^{+} \mathrm{IPC} \forall \forall$. By completeness of $\mathrm{M}^{+} \mathrm{IPC}$ with respect to descriptive frames, there are a descriptive $\mathrm{M}^{+} \mathrm{IPC}$-frame $\mathfrak{F}$ and a valuation $\nu$ on $\mathfrak{F}$ such that $(\mathfrak{F}, \nu) \not=\varphi$. Let $\mathfrak{F}^{\prime}$ be the finite $\mathrm{M}^{+} \mathrm{IPC}$-frame constructed above. Since $t_{0}$ was chosen so that $\widehat{t_{0}}$ refutes $\varphi$ in $\mathfrak{F}$, by Lemma 5.16, $t_{0}$ refutes $\varphi$ in $\mathfrak{F}^{\prime}$. We thus found a finite $\mathrm{M}^{+} I \mathrm{PC}$-frame refuting $\varphi$.

Since $\mathrm{M}^{+} \mathrm{IPC}$ is finitely axiomatizable and has the finite model property, as an immediate corollary to Theorem 5.17, we obtain decidability of $\mathrm{M}^{+} \mathrm{IPC}$, meaning that there is an effective method for determining whether an arbitrary formula is a theorem of $\mathrm{M}^{+}$IPC.

Corollary 5.18. $\mathrm{M}^{+} \mathrm{IPC}$ is decidable.

Remark 5.19. Another consequence of Theorem 5.17 is that $M^{+} I P C$ is the monadic fragment of the intermediate predicate logic of Casari, which is obtained by adding to IQC the Casari formula Cas. This can be seen by utilizing the Translation Theorem of Ono and Suzuki (see [31, Thm. 3.5]).

\section{The Finite MOdel PROPERTY OF $\mathrm{M}^{+} \mathrm{Grz}$}

In this section we prove that $\mathrm{M}^{+} \mathrm{Grz}$ has the finite model property. Our proof, which consists of three steps, is a mixture of selective and standard filtration techniques. The main reasons why the same technique as for $\mathrm{M}^{+} \mathrm{IPC}$ does not work is the lack of persistence in $\mathrm{M}^{+} \mathrm{Grz}$-models and the fact that witnesses for $\forall$-formulas cannot be chosen maximally wrt $Q$-relations. A rough structure of the proof is as follows.

Suppose $\mathrm{M}^{+} \mathrm{Grz} \nvdash \varphi$. Then there is a descriptive $\mathrm{M}^{+} \mathrm{Grz}$-frame $\mathfrak{F}_{0}=\left(W_{0}, R_{0}, E_{0}, P_{0}\right)$ and a valuation $\nu_{0}$ on $W_{0}$ such that $\mathfrak{F}_{0} \not_{0} \varphi$. We build a finite $\mathrm{M}^{+} \mathrm{Grz}$-frame from $\mathfrak{F}_{0}$ in three steps:

(1) First we select a (possibly infinite) partially ordered MS4-frame $\mathfrak{F}_{1}=\left(W_{1}, R_{1}, E_{1}\right)$ from $\mathfrak{F}_{0}$, in which all clusters are clean and $\varphi$ is refuted. An important feature of this step is that $R_{1}$ is not simply the restriction of $R_{0}$ to $W_{1}$, but rather its strengthening. Its construction resembles the construction of $R$-relations from $Q$-relations in the $\rightarrow$-step of the $\mathrm{M}^{+} \mathrm{IPC}$-construction.

(2) Next we construct a (possibly infinite) partially ordered MS4-frame $\mathfrak{F}_{2}$ from $\mathfrak{F}_{1}$, in which all clusters are both clean and finite and $\varphi$ is refuted. In this step we use standard filtration to collapse $E_{1}$ clusters of $\mathfrak{F}_{1}$ so that each cluster contains only one point representing all points that satisfy the same formulas of $\operatorname{Sub}(\varphi)$.

(3) Finally, as in Step 1, we use selective filtration to construct a finite partially ordered MS4-frame $\mathfrak{F}_{3}$ from $\mathfrak{F}_{2}$, in which all clusters are clean (hence $\mathfrak{F}_{3}$ is an $\mathrm{M}^{+} \mathrm{Grz}$-frame) and $\varphi$ is refuted. This step resembles the $\mathrm{M}^{+}$IPC-construction, but in order for $\mathfrak{F}_{3}$ to inherit the bounded cluster size from $\mathfrak{F}_{2}$, we need to add only a single copy of an original point in $\mathfrak{F}_{2}$ to a cluster.

6.1. Step 1: Constructing $\mathfrak{F}_{1}$. Let $\mathfrak{F}_{0}=\left(W_{0}, R_{0}, E_{0}\right)$ be as above. For $x, y \in W_{0}$ let

$$
x \vec{Q}_{0} y \quad \text { iff } \text { there is } w \in W_{0} \text { such that } w \neq x, x R_{0} w \text {, and } w E_{0} y \text {. }
$$

We construct $\mathfrak{F}_{1}=\left(W_{1}, R_{1}, E_{1}\right)$ as follows:

- $W_{1}=\left\{x \in W_{0} \mid x \in \max _{R_{0}} E_{0}(A)\right.$ for some clopen $A$ of $\left.\mathfrak{F}_{0}\right\}$.

- $x R_{1} y \Leftrightarrow x=y$ or $x \vec{Q}_{0} y$ and $x \vDash_{0} \square \psi \Rightarrow y \vDash_{0} \square \psi$ for all $\square \psi \in \operatorname{Sub}(\varphi)$.

- $x E_{1} y \Leftrightarrow x E_{0} y$.

- We define a valuation $\nu_{1}$ on $\mathfrak{F}_{1}$ by $\nu_{1}(p)=\left\{x: x \in \nu_{0}(p)\right\}$ for all $p \in \operatorname{Sub}(\varphi)$, and $\nu_{1}(q)=\varnothing$ for all other propositional variables $q$.

We first show that there is a point in $W_{1}$ which refutes $\varphi$ (in $\mathfrak{F}_{0}$ ). 
Lemma 6.1. There is $v \in W_{0}$ such that $v \not_{0} \varphi\left(R_{0}\right.$-maximally) and $v \in \max _{R_{0}} E_{0}(\nu(\neg \varphi))\left(\right.$ hence $E_{0}(v)$ is clean and $\left.v \in W_{1}\right)$.

Proof. Since $\mathfrak{F}_{0} \not_{0} \varphi$, there is $t \in W_{0}$ such that $t \not_{0} \varphi$. Then $t \in \nu_{0}(\neg \varphi)$, so $t \in E_{0}\left(\nu_{0}(\neg \varphi)\right)$. Because descriptive augmented Kripke frames satisfy $A \in P_{0} \Rightarrow E_{0}(A) \in P_{0}$, we have $E_{0}\left(\nu_{0}(\neg \varphi)\right) \in P_{0}$. Thus, Lemma 2.23 yields $u \in W_{0}$ with $t R_{0} u$ and $u \in \max _{R_{0}} E_{0}\left(\nu_{0}(\neg \varphi)\right)$. Since $u \in E_{0}\left(\nu_{0}(\neg \varphi)\right)$, there is $v \in W_{0}$ with $u E_{0} v$ and $v \not_{0} \varphi$. We now show that $v$ is our desired point. Because $u \in \max _{R_{0}} E_{0}\left(\nu_{0}(\neg \varphi)\right)$, the cluster $E_{0}(u)=E_{0}(v)$ is clean (Lemma 4.8). We show that $v \in \max _{R_{0}} E_{0}\left(\nu_{0}(\neg \varphi)\right.$ ). Suppose $v R_{0} w$ for some $w \in E_{0}\left(\nu_{0}(\neg \varphi)\right)$. By commutativity, there is $u^{\prime}$ such that $u R_{0} u^{\prime}$ and $u^{\prime} E_{0} w$. Then $u^{\prime} \in E_{0}\left(\nu_{0}(\neg \varphi)\right)$, so $u \in \max _{R_{0}} E_{0}\left(\nu_{0}(\neg \varphi)\right)$ implies $u=u^{\prime}$. Thus, $v R_{0} w$ and $v E_{0} w$, yielding that $v=w$ as $v$ is in a clean cluster. Now, since $v \in \max _{R_{0}} E_{0}\left(\nu_{0}(\neg \varphi)\right)$ and $v \in \nu_{0}(\neg \varphi)$, it is easy to see that $v$ is $R_{0}$-maximal with respect to $\varphi$, hence is our desired point.

We next highlight some fundamental properties of $\mathfrak{F}_{1}$.

\section{Lemma 6.2.}

(1) $E_{0}(x) \subseteq W_{0}$ is a clean cluster in $\mathfrak{F}_{0}$ for all $x \in W_{1}$.

(2) If $x \in W_{1}$, then $E_{0}(x) \subseteq W_{1}$.

(3) $x \vec{Q}_{0} y$ iff $x Q_{0} y$ but $x E_{0} y$ for all $x, y \in W_{1}$.

(4) The restriction of $\vec{Q}_{0}$ to $W_{1}$ is a strict partial order.

(5) $R_{1}$ is a partial order.

(6) $E_{1}$ is an equivalence relation.

(7) $R_{1}$ and $E_{1}$ satisfy commutativity.

(8) $\mathfrak{F}_{1}$ has clean clusters.

(9) For $x \in W_{1}$ and $\square \gamma \in \operatorname{Sub}(\varphi)$, if $x \forall_{0} \square \gamma$, then there is $y \in W_{1}$ such that $x R_{1} y, y \in A \cap \max _{R_{0}} E_{0}(A)$, where

$$
A=\nu_{0}(\neg \square \gamma) \cap \bigcap\left\{\nu_{0}(\square \psi) \mid \square \psi \in \operatorname{Sub}(\varphi) \text { and } x \vDash_{0} \square \psi\right\},
$$

and $y \forall_{0} \square \gamma R_{0}$-maximally, hence $y \not_{0} \gamma$.

Proof. (1) This is an immediate consequence of Lemma 4.8

(2) Let $x \in W_{1}$ and $y \in E_{0}(x)$. Then $x \in \max _{R_{0}} E(A)$ for some clopen $A \subseteq W_{0}$. Therefore, $E_{0}(x)$ is clean by (1), and so $y \in \max _{R_{0}} E(A)$ by Lemma 4.7(1). Thus, $y \in W_{1}$.

(3) The implication from right to left is obvious. For the converse, suppose that $x, y \in W_{1}$ and there is $w \in W_{0}$ such that $w \neq x, x R_{0} w$, and $w E_{0} y$. Then clearly $x Q_{0} y$. Also, since $x$ is from a clean cluster, $x E_{0} w$. Thus, $x E_{0} y$.

(4) Irreflexivity of $\overrightarrow{Q_{0}}$ on $W_{1}$ follows from the reflexivity of $E_{0}$ and (3). We show that $\vec{Q}_{0}$ is transitive on $W_{1}$. Suppose $x \overrightarrow{Q_{0}} y \overrightarrow{Q_{0}} z$ for $x, y, z \in W_{1}$. Then there are $y^{\prime} \neq x$ and $z^{\prime} \neq y$ with $x R_{0} y^{\prime}, y^{\prime} E_{0} y$ and $y R_{0} z^{\prime}$ and $z^{\prime} E_{0} z$. By commutativity, there is $z^{\prime \prime}$ with $y^{\prime} R_{0} z^{\prime \prime}$ and $z^{\prime \prime} E_{0} z$. Therefore, $x R_{0} z^{\prime \prime}$ and $z^{\prime \prime} E_{0} z$. If we had $x=z^{\prime \prime}$, then we would obtain $x R_{0} y^{\prime} R_{0} x$, and so $x=y^{\prime}$ by Lemma $4.7(2)$. The latter contradicts the choice of $y^{\prime}$. Thus, $z^{\prime \prime} \neq x$ and so $x \overrightarrow{Q_{0}} z$.

(5) $R_{1}$ is reflexive by definition. To see that $R_{1}$ is transitive, suppose $x, y, z \in W_{1}$ with $x R_{1} y R_{1} z$. Without loss of generality we may assume that $x, y, z$ are pairwise distinct. Then $x \overrightarrow{Q_{0}} y$ and $y \overrightarrow{Q_{0}} z$, so $x \overrightarrow{Q_{0}} z$ by (3). Moreover, if $x \models \square \psi$ for $\square \psi \in \operatorname{Sub}(\varphi)$, then since $x R_{1} y R_{1} z$, we have $y \models \square \psi$ and so $z \models \square \psi$. Therefore, $R_{1}$ is transitive. Finally, if $x R_{1} y R_{1} x$ and $x \neq y$, then $x \vec{Q}_{0} y \vec{Q}_{0} x$. The latter implies $x \overrightarrow{Q_{0}} x$ by transitivity of $\overrightarrow{Q_{0}}$, which contradicts irreflexivity of $\overrightarrow{Q_{0}}$. Thus, $R_{1}$ is anti-symmetric.

(6) This is immediate since $E_{1}$ is an equivalence relation.

(7) Suppose that $x R_{1} y$ and $x E_{1} z$. Without loss of generality we may assume that $x \neq y$ and $x \neq z$. Then $x \vec{Q}_{0} y$, so there is $u \in W_{0}$ such that $x \neq u, x R_{0} u$, and $u E_{0} y$. By commutativity in $W_{0}$, there is $v$ such that $z R_{0} v$ and $v E_{0} u$. We show that $v$ is the required witness for commutativity in $W_{1}$. From $v E_{0} u$ and $u E_{0} y$ we have $v E_{0} y$, so $v \in W_{1}$ by (2). Because $x \neq u, x R_{0} u$, and $x$ is from a clean cluster, we have $x E_{0} u$. Thus, $z E_{0} v$. In particular, $z \neq v$, and so $z \overrightarrow{Q_{0}} v$. Moreover, $z R_{0} v$ gives that if $z \vDash_{0} \square \gamma$, then $v \vDash_{0} \square \gamma$, so $z R_{1} v$. From $v E_{0} y$ we have $v E_{1} y$, yielding commutativity in $W_{1}$. 
(8) Suppose there are $x, y \in W_{1}$ with $x \neq y, x E_{1} y$, and $x R_{1} y$. Since $x E_{1} y$, we have $x E_{0} y$, and because $x R_{1} y$ and $x \neq y$, we have $x \vec{Q}_{0} y$. Thus, there is $w \in W_{0}$ with $x \neq w, x R_{0} w$, and $w E_{0} y$. From $x E_{0} y$ and $y E_{0} w$ we have $x E_{0} w$. By (1), $x$ is chosen from a clean cluster in $W_{0}$, so $x R_{0} w$ and $x E_{0} w$ imply $x=w$, a contradiction.

(9) Suppose $x \not_{0} \square \gamma$. Consider

$$
A=\nu_{0}(\neg \square \gamma) \cap \bigcap\left\{\nu_{0}(\square \psi) \mid \square \psi \in \operatorname{Sub}(\varphi) \text { and } x \vDash_{0} \square \psi\right\} .
$$

Clearly $x \in A$, so $x \in E_{0}(A)$. We have $x \in \max _{R_{0}} E_{0}(A)$ or $x \notin \max _{R_{0}} E_{0}(A)$.

Case 1: $x \in \max _{R_{0}} E_{0}(A)$

If $x \in \max _{R_{0}} E_{0}(A)$, then from $x R_{0} w$ and $x \neq w$ it follows that $w \notin E_{0}(A)$, so $w \notin A$. But $x R_{0} w$ implies $w \in \bigcap\left\{\nu_{0}(\square \psi) \mid \square \psi \in \operatorname{Sub}(\varphi)\right.$ and $\left.x \vDash_{0} \square \psi\right\}$, so we must have $w \notin \nu_{0}(\neg \square \gamma)$. Therefore, $w \vDash_{0} \square \gamma$. Since $x \forall_{0} \square \gamma$ but $w \vDash_{0} \square \gamma$ for all $w \neq x$ with $x R_{0} w$, we must have $x \forall_{0} \square \gamma R_{0}$-maximally. Thus, $x \forall_{0} \gamma$, and $x$ is our desired point.

Case 2: $x \notin \max _{R_{0}} E_{0}(A)$

If $x \notin \max _{R_{0}} E_{0}(A)$, then Lemma 2.26(2) yields $t \in \max _{R_{0}} E_{0}(A)$ such that $x \neq t$ and $x R_{0} t$. But then $t E_{0} y$ for some $y \in A$. Since $t \in \max _{R_{0}} E_{0}(A)$, we have $t \in W_{1}$, so $y \in W_{1}$ by (2). From $x \neq t$ and $x R_{0} t$ it follows that $x \overrightarrow{Q_{0}} y$. By the choice of $y \in A$, if $x \vDash_{0} \square \psi$ then $y \vDash_{0} \square \psi$ for all $\square \psi \in \operatorname{Sub}(\varphi)$, so $x R_{1} y$. Since $y \in A$, we have $y \not_{0} \square \gamma$. To see that $y \not_{0} \square \gamma R_{0}$-maximally, suppose $y R_{0} z$ and $z \nvdash_{0} \square \gamma$. If $x \vDash_{0} \square \psi$, then $y \vDash_{0} \square \psi$ (as $y \in A$ ), so $y R_{0} z$ implies $z \vDash_{0} \square \psi$. Thus, $z \in A$, hence $z \in E_{0}(A)$, and maximality of $y$ in $E_{0}(A)$ yields $y=z$. Consequently, $y$ is $R_{0}$-maximal with respect to $\square \gamma$.

We conclude Step 1 by proving the truth lemma for $\mathfrak{F}_{1}$.

Lemma 6.3 (Truth Lemma). For $x \in W_{1}$ and $\psi \in \operatorname{Sub}(\varphi)$,

$$
\left(\mathfrak{F}_{0}, x\right) \vDash_{0} \psi \Leftrightarrow\left(\mathfrak{F}_{1}, x\right) \vDash_{1} \psi \text {. }
$$

Proof. The proof is by induction on the complexity of $\psi$. The base case $\psi=p$ is clear from the definition of $\nu_{1}$. The cases of $\psi=\psi_{1} \wedge \psi_{2}$ and $\psi=\neg \psi_{1}$ are straightforward, so we focus on the cases $\psi=\forall \psi_{1}$ and $\psi=\square \psi_{1}$.

Suppose $\psi=\forall \psi_{1}$. If $x \not_{0} \forall \psi_{1}$, then $x E_{0} y$ for some $y \not_{0} \psi_{1}$. By Lemma 6.2(2), $y \in W_{1}$, so $y \not_{1} \psi_{1}$ by the inductive hypothesis. From $x E_{0} y$ we have $x E_{1} y$ by the definition of $E_{1}$. Thus, $x \forall_{1} \forall \psi_{1}$. The proof of the converse implication is immediate.

Suppose $\psi=\square \psi_{1}$. If $x \not_{0} \square \psi_{1}$, then by Lemma 6.2(9), there is $y \in W_{1}$ such that $x R_{1} y$ and $y \not_{0} \psi_{1}$. By the inductive hypothesis, $y \nvdash_{1} \psi_{1}$, hence $x \nvdash_{1} \square \psi_{1}$. Conversely, if $x \nvdash_{1} \square \psi_{1}$, then there is $y \in W_{1}$ such that $x R_{1} y$ and $y \not_{1} \psi_{1}$. By the inductive hypothesis, $y \not_{0} \psi_{1}$. If $x=y$, then $x \forall_{0} \psi_{1}$, hence $x \nvdash_{0} \square \psi_{1}$. If $x \neq y$, then as $x R_{1} y$, we have $x \vec{Q}_{0} y$ and $x \vDash_{0} \square \gamma$ implies $y \vDash_{0} \square \gamma$ for all $\square \gamma \in \operatorname{Sub}(\varphi)$. Since $y \not_{0} \psi_{1}$, we have $y \not_{0} \square \psi_{1}$. Thus, $x \not_{0} \square \psi_{1}$.

6.2. Step 2: Constructing $\mathfrak{F}_{2}$. In this step we use the standard filtration technique to construct $\mathfrak{F}_{2}$ from $\mathfrak{F}_{1}$ by 'collapsing' $E_{1}$-clusters into finitely many classes. Thus, $\mathfrak{F}_{2}$ will have finitely many clusters.

Define an equivalence relation $\sim$ on $W_{1}$ by

$$
x \sim y \Leftrightarrow\left(x E_{1} y \text { and } x \vDash_{1} \gamma \Leftrightarrow y \vDash_{1} \gamma \text { for all } \gamma \in \operatorname{Sub}(\varphi)\right) .
$$

We construct $\mathfrak{F}_{2}=\left(W_{2}, R_{2}, E_{2}\right)$ as follows:

- $W_{2}=W_{1} / \sim=\left\{[x]: x \in W_{1}\right\}$ where $[x]$ denotes the $\sim$-equivalence class of $x$.

- For $[x],[y] \in W_{2},[x] R_{2}[y] \Leftrightarrow[x]=[y]$ or $x R_{1} y$.

- For $[x],[y] \in W_{2},[x] E_{2}[y] \Leftrightarrow x E_{1} y$.

- $\nu_{2}(p)=\left\{[x]: x \in \nu_{1}(p)\right\}$ for all $p \in \operatorname{Sub}(\varphi)$, and $\nu_{2}(q)=\varnothing$ for all other propositional variables $q$.

Lemma 6.4. The relations $E_{2}$ and $R_{2}$ are well defined, and so is the valuation $v_{2}$.

Proof. It is easy to see that $E_{2}$ and $v_{2}$ are well defined. We show that $R_{2}$ is well defined. Let $x, y, x^{\prime}, y^{\prime} \in W_{1}$ with $x \sim x^{\prime}, y \sim y^{\prime}$, and $[x] R_{2}[y]$. Then $[x]=[y]$ or $x R_{1} y$. If $[x]=[y]$, we have $\left[x^{\prime}\right]=[x]=[y]=\left[y^{\prime}\right]$, and so $\left[x^{\prime}\right] R_{2}\left[y^{\prime}\right]$. If $x R_{1} y$, then $x=y$ or $x \overrightarrow{Q_{0}} y$ and $x \vDash_{0} \square \gamma$ implies $y \vDash_{0} \square \gamma$ for all $\square \gamma \in \operatorname{Sub}(\varphi)$. The former case implies $[x]=[y]$ which we have already considered. In the latter case, from $x \vec{Q}_{0} y$ it follows that 
$x Q_{0} y$ and $x E_{0} y$ by Lemma 6.2(3). Note that $x^{\prime} \sim x$ implies $x^{\prime} E_{1} x$ and so $x^{\prime} E_{0} x$. Similarly, $y^{\prime} E_{0} y$. By transitivity of $Q_{0}$ we thus have $x^{\prime} Q_{0} y^{\prime}$. Moreover, $x^{\prime} E_{0} x, y^{\prime} E_{0} y$, and $x E_{0} y$ imply that $x^{\prime} \mathscr{H}_{0} y^{\prime}$. Thus, $x^{\prime} \vec{Q}_{0} y^{\prime}$ by Lemma 6.2(3). If $\square \gamma \in \operatorname{Sub}(\varphi)$ and $x^{\prime} \vDash_{0} \square \gamma$, then $x \vDash_{0} \square \gamma$ since $x^{\prime} \sim x$. So $y \vDash_{0} \square \gamma$ by assumption. But then $y^{\prime} \vDash_{0} \square \gamma$ since $y^{\prime} \sim y$. This shows that $x^{\prime} R_{1} y^{\prime}$, so $\left[x^{\prime}\right] R_{2}\left[y^{\prime}\right]$.

In the following lemma we highlight some properties of $\mathfrak{F}_{2}$.

\section{Lemma 6.5.}

(1) $R_{2}$ is a partial order.

(2) $E_{2}$ is an equivalence relation.

(3) $R_{2}$ and $E_{2}$ satisfy commutativity.

(4) $\mathfrak{F}_{2}$ has clean clusters.

(5) For $[x] \in W_{2},\left|E_{2}([x])\right| \leq 2^{n}$, where $n=|\operatorname{Sub}(\varphi)|$.

(6) For $[x] \in W_{2}$ and $\square \gamma \in \operatorname{Sub}(\varphi)$, if $x \not_{1} \square \gamma$, then there is $[y] \in W_{2}$ such that $[x] R_{2}[y]$ and $y \forall_{1} \square \gamma$ $R_{1}$-maximally.

Proof. (1) Reflexivity of $R_{2}$ is immediate from the definition, and transitivity and antisymmetry follow from transitivity and antisymmetry of $R_{1}$.

(2) This follows from $E_{1}$ being an equivalence relation.

(3) This follows from $R_{1}$ and $E_{1}$ satisfying commutativity.

(4) Suppose there are $[x] \neq[y]$ in $W_{2}$ with $[x] R_{2}[y]$ and $[x] E_{2}[y]$. Then $x \neq y$, so by the definition of $R_{2}$ and $E_{2}$, we have $x R_{1} y$ and $x E_{1} y$ which yields a dirty cluster in $\mathfrak{F}_{1}$, contradicting Lemma $6.2(8)$.

(5) This follows from the fact that there are at most $2^{n} \sim$-equivalence classes in each cluster (see, e.g., [10, Prop. 5.24]).

(6) Suppose $x \not \not_{1} \square \gamma$. By Lemma 6.3, $x \not \not_{0} \square \gamma$, so by Lemma 6.2(9), there is $y \in W_{1}$ such that $x R_{1} y$, $y \in A \cap \max _{R_{0}} E_{0}(A)$, and $y \forall_{0} \square \gamma R_{0}$-maximally, where

$$
A=\nu_{0}(\neg \square \gamma) \cap \bigcap\left\{\nu_{0}(\square \psi) \mid \square \psi \in \operatorname{Sub}(\varphi) \text { and } x \vDash_{0} \square \psi\right\} .
$$

Then $[x] R_{2}[y]$ and by Lemma 6.3, $y \not_{1} \square \gamma$. We show that $y$ is $R_{1}$-maximal with respect to $\square \gamma$. Suppose $y R_{1} z$ and $z \not \not_{1} \square \gamma$. By Lemma $6.3 .3 \not \not_{0} \square \gamma$, and from $y R_{1} z$ it follows that $y=z$ or $y \vec{Q}_{0} z$ and $y \vDash_{0} \square \psi$ implies $z \vDash_{0} \square \psi$ for all $\square \psi \in \operatorname{Sub}(\varphi)$. Suppose the latter. Since $z \not \nvdash_{0} \square \gamma$, we have $z \in \nu_{0}(\neg \square \gamma)$. If $x \vDash_{0} \square \psi$ for $\square \psi \in \operatorname{Sub}(\varphi)$, then $y \in A$ implies $y \vDash_{0} \square \psi$. So $y R_{1} z$ then gives $z \vDash_{0} \square \psi$. Therefore, $z \in \bigcap\left\{\nu_{0}(\square \psi) \mid \square \psi \in \operatorname{Sub}(\varphi)\right.$ and $\left.x \vDash_{0} \square \psi\right\}$, and hence $z \in A$. As $y \overrightarrow{Q_{0}} z$, there is $w \in W_{0}$ such that $y \neq w$, $y R_{0} w$, and $w E_{0} z$. Then $w \in E_{0}(A)$, and maximality of $y$ in $E_{0}(A)$ yields $y=w$, contradicting $y \neq w$. Thus, $y=z$, and so $y$ is $R_{1}$-maximal with respect to $\square \gamma$.

We conclude Step 2 by showing the truth lemma for $\mathfrak{F}_{2}$.

Lemma 6.6 (Truth Lemma). For $[x] \in W_{2}$ and $\psi \in \operatorname{Sub}(\varphi)$,

$$
\left(\mathfrak{F}_{1}, x\right) \vDash_{1} \psi \Leftrightarrow\left(\mathfrak{F}_{2},[x]\right) \vDash_{2} \psi .
$$

Proof. The proof is by induction on the complexity of $\psi$. The base case $\psi=p$ follows from the definition of $\nu_{2}$. The cases of $\psi=\psi_{1} \wedge \psi_{2}$ and $\psi=\neg \psi_{1}$ are straightforward, and the $\forall$-case follows from the definition of $E_{2}$. Suppose that $\psi=\square \psi_{1}$. If $x \not_{1} \square \psi_{1}$, then there is $y \in W_{1}$ with $x R_{1} y$ and $y \not \not_{1} \psi_{1}$. Therefore, $[x] R_{2}[y]$ and $[y] \forall_{2} \psi_{1}$ by the inductive hypothesis. Thus, $[x] \not_{2} \square \psi_{1}$. Conversely, if $[x] \forall_{2} \square \psi_{1}$, then there is $y \in W_{1}$ with $[x] R_{2}[y]$ and $[y] \not_{2} \psi_{1}$. By the inductive hypothesis, $y \nvdash_{1} \psi_{1}$. If $[x]=[y]$, then $x \nvdash_{1} \square \psi_{1}$ by definition of $\sim$. If $[x] \neq[y]$, then $x R_{1} y$ and again $x \not \not_{1} \square \psi_{1}$.

6.3. Step 3: Constructing $\mathfrak{F}_{3}$. We are ready for our final step, in which we construct $\mathfrak{F}_{3}=\left(W_{3}, R_{3}, E_{3}\right)$ by selective filtration from $\mathfrak{F}_{2}$. This is done by constructing a sequence of finite partially ordered MS4-frames $\mathfrak{F}_{3 . h}=\left(W_{3 . h}, R_{3 . h}, E_{3 . h}\right)$ with clean clusters so that $\mathfrak{F}_{3 . h} \subseteq \mathfrak{F}_{3 . h+1}$ for all $h<\omega$. We then show that this construction eventually terminates.

Similar to the construction for $\mathrm{M}^{+}$IPC, for each point $[x] \in W_{2}$ that we select, we create a copy of the point, give it a new name, say $t$, and let $\widehat{t}=[x]$ denote the original point in $W_{2}$ that $t$ represents and will behave similar to. However, we take a bit more care with the copies in this construction than in the 
construction for $\mathrm{M}^{+} \mathrm{IPC}$. In particular, we will never create two copies of the same original point within one cluster. This will ensure that the cluster size in $\mathfrak{F}_{3}$ has the same bound as the cluster size in $\mathfrak{F}_{2}$.

Before we begin the construction, we highlight an important property we will need for selecting our points.

Lemma 6.7. For $[x] \in W_{2}$ and $\square \gamma \in \operatorname{Sub}(\varphi)$, if $[x] \not \not_{2} \square \gamma$, then there is $[y] \in W_{2}$ such that $[x] R_{2}[y]$ and $[y] \not \not_{2} \square \gamma R_{2}$-maximally.

Proof. Suppose $[x] \not \nvdash_{2} \square \gamma$. By Lemma 6.6, $x \not \not_{1} \square \gamma$, and by Lemma 6.5(6), there is $[y] \in W_{2}$ such that $[x] R_{2}[y]$ and $y \not \not_{1} \square \gamma R_{1}$-maximally. Applying Lemma 6.6 again yields $[y] \not_{2} \square \gamma$. To see that $[y]$ is $R_{2^{-}}$ maximal with respect to $\square \gamma$, suppose $[y] R_{2}[z]$ and $[z] \not_{2} \square \gamma$. By definition of $R_{2}$, either $[y]=[z]$ or $y R_{1} z$. If $y R_{1} z$, then by $R_{1}$-maximality of $y$, we have $y=z$, so $[y]=[z]$, and hence [y] must be $R_{2}$-maximal with respect to $\square \gamma$.

Throughout the construction, for each $t \in W_{3 . h}$, we associate the following sets of subformulas:

$$
\begin{aligned}
\Sigma^{\forall}(t) & =\left\{\forall \delta \in \operatorname{Sub}(\varphi) \mid \widehat{t} \not_{2} \forall \delta\right\} \\
\Sigma^{\square}(t) & =\left\{\square \gamma \in \operatorname{Sub}(\varphi) \mid \widehat{t} \not_{2} \square \gamma, \widehat{t} \vDash_{2} \gamma\right\} .
\end{aligned}
$$

We start with $\mathfrak{F}_{3.0}=\left(W_{3.0}, R_{3.0}, E_{3.0}\right)$ where

$$
W_{3.0}=\left\{t_{0}\right\}, \quad R_{3.0}=W_{3.0}^{2}, \quad E_{3.0}=W_{3.0}^{2}
$$

and $\widehat{t_{0}}=\left[x_{0}\right] \in W_{2}$ is a point with $\left[x_{0}\right] \not \not_{2} \varphi$. This will be a root of our frame and has $Q_{3}$-depth 1 . Let $W_{3 .-1}=R_{3 .-1}=E_{3 .-1}=\varnothing$. Suppose $\mathfrak{F}_{3 . h-1}=\left(W_{3 . h-1}, R_{3 . h-1}, E_{3 . h-1}\right)$ has already been constructed and is a partially ordered MS4-frame with clean clusters. We construct $\mathfrak{F}_{3 . h}$ by the following steps.

Step $\forall$ (Horizontal): Let $W_{3 . h}^{\forall}=W_{3 . h-1}, R_{3 . h}^{\forall}=R_{3 . h-1}$, and $E_{3 . h}^{\forall}=E_{3 . h-1}$. For each cluster $E_{3 . h}(t) \subseteq$ $W_{3 . h-1} \backslash W_{3 . h-1}^{\forall}$, consider $\forall \delta \in \Sigma^{\forall}(t)$. If there is no $s \in W_{3 . h}^{\forall}$ already such that $t E_{3 . h}^{\forall} s$ and $\widehat{s} \forall_{2} \delta$, we add a witness to our new frame as follows. Since $\widehat{t} \not \not_{2} \forall \delta$, there exists $[x] \in W_{2}$ such that $\widehat{t} E_{2}[x]$ and $[x] \not_{2} \delta$. We add the point $s$ to $W_{3 . h}^{\forall}$ where $\widehat{s}=[x](s$ is a distinct new copy of $[x])$, the relations $(s, s)$ to $R_{3 . h}^{\forall}$, the relations $(t, s)$ to $E_{3 . h}^{\forall}$ and generate the least equivalence relation.

Step $\square$ (Vertical): Let $W_{3 . h}^{\square}=W_{3 . h}^{\forall}, R_{3 . h}^{\square}=R_{3 . h}^{\forall}$, and $E_{3 . h}^{\square}=E_{3 . h}^{\forall}$. For $t \in W_{3 . h}^{\forall} \backslash W_{3 . h-1}^{\forall}$ (hence including any points added in the horizontal step), consider $\square \gamma \in \Sigma \square(t)$ where $\widehat{t} \not_{2} \square \gamma$, but $\widehat{t} \vDash_{2} \gamma$ (thus, $t$ isn't witnessing the formula $\square \gamma$ itself), and there is no $s \in W_{3 . h}^{\square}$ already such that $t R_{3 . h}^{\square} s$ and $\widehat{s} \not \nvdash_{2} \square \gamma R_{2^{-}}$ maximally (such an $s$ could have been added in a previous stage to satisfy commutativity). For each such $\square \gamma$, since $\widehat{t} \not_{2} \square \gamma$ and $\hat{t}=[w]$ for some $[w] \in W_{2}$, we have $[w] \not \not_{2} \square \gamma$. By Lemma 6.7 there is $[x] \in W_{2}$ such that $[w] R_{2}[x]$ and $[x]$ is $R_{2}$-maximal with respect to $\square \gamma$. We add the point $s$ to $W_{3 . h}^{\square}$ where $\widehat{s}=[x]$, $(t, s)$ and $(s, s)$ to $R_{3 . h}^{\square}$ and close under transitivity, and add $(s, s)$ to $E_{3 . h}^{\square}$. To make sure commutativity is satisfied, for each $w \in E_{3 . h}^{\square}(t)$, if there is already $s_{w} \in E_{3 . h}^{\square}(s)$ such that $\widehat{w} R_{2} \widehat{s_{w}}$, we simply add the relation $\left(w, s_{w}\right)$ to $R_{3 . h}^{\square}$. If there is no such $s_{w}$, then by commutativity in $W_{2}$, there is $\left[x_{w}\right] \in W_{2}$ such that $\widehat{w} R_{2}\left[x_{w}\right]$ and $\left[x_{w}\right] E_{2}[x]$, so we add $s_{w}$ to $W_{3 . h}^{\square}$, where $\widehat{s_{w}}=\left[x_{w}\right]$. We then add $\left(w, s_{w}\right)$ to $R_{3 . h}^{\square}$ and close it under reflexivity and transitivity, and add $\left(s_{w}, s\right)$ to $E_{3 . h}^{\square}$ and generate the smallest equivalence relation.

To end this stage of the construction, we let $\mathfrak{F}_{3 . h}=\left(W_{3 . h}, R_{3 . h}, E_{3 . h}\right)$ where

$$
W_{3 . h}=W_{3 . h}^{\square}, R_{3 . h}=R_{3 . h}^{\square} \text { and } E_{3 . h}=E_{3 . h}^{\square} .
$$

Lemma 6.8. $\mathfrak{F}_{3 . h}$ is a finite partially ordered MS4-frame with clean clusters.

Proof. In the $\forall$-step we only added reflexive arrows to $R_{3 . h}^{\forall}$, so $R_{3 . h}^{\forall}$ is a partial order. In the $\square$-step we close $R_{3 . h}^{\square}$ under reflexivity and transitivity each time we add a new arrow, so $R_{3 . h}^{\square}$ is reflexive and transitive. Moreover, we we only add $R_{3 . h}^{\square}$ arrows from points that were already present in $W_{3 . h}^{\forall}$ into points that are freshly added in the $\square$-step of round $h$. Thus, $R_{3 . h}^{\square}$ is antisymmetric. That $E_{3 . h}$ is an equivalence relation and that $\mathfrak{F}_{3 . h}$ satisfies commutativity follow from the construction. Finally, to see that $\mathfrak{F}_{3 . h}$ has only clean clusters, note that in the $\forall$-step all freshly introduced $E_{h}$-relations are of the shape $(s, t)$ where $s$ or $t \in W_{3 . h}^{\forall} \backslash W_{3 . h-1}$. Since no non-reflexive $R_{h}$-arrows are introduced in this step, no dirty cluster could have been built. We have already discussed the shape of the $R_{h}$ arrows introduced in the $\square$-step. This guarantees that no cluster in $W_{3 . h}^{\forall}$ is made dirty. The freshly introduced $E_{h}$-relations in these steps are of the shape 
$(s, t)$ where $s, t \in W_{3 . h}^{\square} \backslash W_{3 . h}^{\forall}$. Since no non-reflexive $R_{h}$-relations exist between these points, we infer that all clusters are clean.

The following lemma summarizes some useful properties of $\mathfrak{F}_{3}$. In the following let

$$
n=|\operatorname{Sub}(\varphi)| \text { and } m=|\{\square \psi: \square \psi \in \operatorname{Sub}(\varphi)\}| \text {. }
$$

Lemma 6.9. Let $t, u \in W_{3 . h}$.

(1) If $t E_{3 . h} u$, then $\widehat{t} E_{2} \widehat{u}$.

(2) If $t E_{3 . h} u$, then $\Sigma^{\forall}(t)=\Sigma^{\forall}(u)$. (This ensures that we only need to perform the $\forall$-step once per cluster).

(3) If $t E_{3 . h} u$ and $t \neq u$, then $\widehat{t} \neq \widehat{u}$. (This ensures that one cluster does not contain two different copies of the same point, so our cluster size remains bounded).

(4) If $[t] \overrightarrow{Q_{2}}[u]$, then $t \vec{Q}_{0} u$.

(5) If $t R_{3 . h} u$, then $\widehat{t} R_{2} \widehat{u}$.

(6) If $t \overrightarrow{Q_{3 . h}} u$, then $\widehat{t} \overrightarrow{Q_{2}} \widehat{u}$. Thus, if $t \overrightarrow{Q_{3 . h}} u$, then $\widehat{t} \neq \widehat{u}$.

(7) A formula $\square \gamma \in \operatorname{Sub}(\varphi)$ can be witnessed at most $2^{n}$ times in clusters along an $R_{3 . h}$-chain. (This shows that $\square \gamma$ can be witnessed at most $2^{n}$ times per $Q_{3 . h}$-chain.)

Proof. (1) This follows from the construction.

(2) By (1), $t E_{3 . h} u$ implies $\widehat{t} E_{2} \widehat{u}$, so $\widehat{t} \vDash_{2} \forall \gamma$ iff $\widehat{u} \vDash_{2} \forall \gamma$.

(3) Suppose $t E_{3 . h} u$ and $\widehat{t}=\widehat{u}$, and without loss of generality assume that $t$ was added to the cluster before $u$, so either $u$ is added to witness some formula $\forall \delta_{i}$ where $\widehat{u} \nvdash_{2} \delta_{i}$, or $u$ is added as a commutativity witness for some point from the cluster below. However, by construction, $u$ would not have been added to witness a formula $\forall \delta_{i}$, because if $\widehat{u} \not_{2} \delta_{i}$, then $\widehat{t}=\widehat{u}$ implies that $\widehat{t} \forall_{2} \delta_{i}$, so $t$ is already a viable witness in the cluster for any such formula, contradicting the $\forall$-step of the construction. Furthermore, $u$ would not be added as a commutativity witness for some point $w$ in the cluster immediately below, because then in $W_{2}$ we would have $\widehat{w} R_{2} \widehat{u}$, so $\widehat{w} R_{2} \widehat{t}$, and a new $R_{3 . h}$-relation would have been added from $w$ to $t$ instead, contradicting the $\square$-step of the construction. Thus, we must have $\widehat{t} \neq \widehat{u}$.

(4) Suppose $[t] \overrightarrow{Q_{2}}[u]$. Then there is $[w] \in W_{2}$ with $[t] \neq[w],[t] R_{2}[w]$, and $[w] E_{2}[u]$. From the definitions of $E_{1}$ and $E_{2},[w] E_{2}[u]$ implies $w E_{0} u$. By definition of $R_{2},[t] R_{2}[w]$ and $[t] \neq[w]$ imply $t R_{1} w$. Since $[t] \neq[w]$, we have $t \neq w$, so $t \vec{Q}_{0} w$ by the definition of $R_{1}$. Then there is $v \in W_{1}$ with $t \neq v, t R_{0} v$, and $v E_{0} w$. Since $v E_{0} w$, we have $v E_{0} u$. Thus, $t \neq v, t R_{0} v$, and $v E_{0} u$, and hence $t \vec{Q}_{0} u$.

(5) This follows from the construction.

(6) If $t \overrightarrow{Q_{3 . h}} u$, then there is $w$ such that $t \neq w, t R_{3 . h} w$, and $w E_{3 . h} u$. By (5), $\widehat{t} R_{2} \widehat{w}$ and $\widehat{w}$ must come from a different cluster in $W_{2}$ than $\widehat{t}$, so $\widehat{t} \neq \widehat{w}$. We also have $\widehat{w} E_{2} \widehat{u}$ by $(1)$, so $\widehat{t} Q_{2} \widehat{u}$. Because $\mathfrak{F}_{2}$ has clean clusters, we must have $\widehat{t} \neq \widehat{u}$.

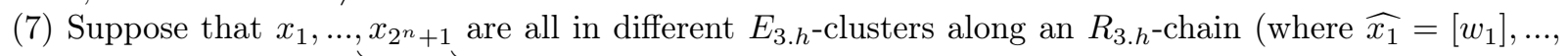
$\left.\widehat{x}_{2^{n}+1}=\left[w_{2^{n}+1}\right]\right)$, so $x_{1} \overrightarrow{Q_{3 . h}} \ldots \overrightarrow{Q_{3 . h}} x_{2^{n}+1}$, and all have been added to witness a formula $\square \gamma \in \operatorname{Sub}(\varphi)$. Thus, $\widehat{x_{i}} \not_{2} \square \gamma R_{2}$-maximally for $i=1, \ldots, 2^{n}+1$. Because there are only $2^{n}$ subsets of Sub $(\varphi)($ where $n=|\operatorname{Sub}(\varphi)|)$, the pigeonhole principle implies that there are some $i$ and $j$ with $i \neq j$ (assume $i<j$ ) where $\widehat{x_{i}}$ and $\widehat{x_{j}}$ satisfy the same subformulas of $\varphi$. By $(6), \widehat{x_{i}} \overrightarrow{Q_{2}} \widehat{x_{j}}$ and $\widehat{x_{i}} \neq \widehat{x_{j}}$. If $\widehat{x_{i}} R_{2} \widehat{x_{j}}$, then $R_{2}$-maximality of $\widehat{x_{i}}$ with respect to $\square \gamma$ implies $\widehat{x_{i}}=\widehat{x_{j}}$, contradicting $\widehat{x_{i}} \neq \widehat{x_{j}}$, so we must have $\widehat{x_{i}} R_{2} \widehat{x_{j}}$ and hence $\left[w_{i}\right] R_{2}\left[w_{j}\right]$. Since $\widehat{x_{i}} \overrightarrow{Q_{2}} \widehat{x_{j}}$ we have $\left[w_{i}\right] \vec{Q}_{2}\left[w_{j}\right]$. By (4), we then have $w_{i} \vec{Q}_{0} w_{j}$. Since $\left[w_{i}\right]$ and $\left[w_{j}\right]$ satisfy the same formulas in $\operatorname{Sub}(\varphi)$, we have $\left[w_{i}\right] \vDash_{2} \square \beta \Leftrightarrow\left[w_{j}\right] \vDash_{2} \square \beta$ for $\square \beta \in \operatorname{Sub}(\varphi)$. By Lemmas 6.6 and 6.3. $w_{i} \vDash_{0} \square \beta \Leftrightarrow w_{j} \vDash_{0} \square \beta$. Thus, $w_{i} R_{1} w_{j}$ and hence $\left[w_{i}\right] R_{2}\left[w_{j}\right]$, a contradiction.

We now prove that the end result of our construction is a finite frame, using the definitions of bounded cluster size, bounded $R$-branching, and bounded $R$-depth given in Definition 5.10 .

Lemma 6.10. $\mathfrak{F}_{3 . h}=\left(W_{3 . h}, R_{3 . h}, E_{3 . h}\right)$ has cluster size bounded by $2^{n}$ for all $h<\omega$.

Proof. By Lemma 6.5(5), the cluster size in $\mathfrak{F}_{2}$ is bounded by $2^{n}$, and by Lemma 6.9 (3), we do not add copies of the same points to a cluster in $\mathfrak{F}_{3 . h}$. Thus, cluster size in $\mathfrak{F}_{3 . h}$ is bounded by $2^{n}$. 
Lemma 6.11. $\mathfrak{F}_{3 . h}=\left(W_{3 . h}, R_{3 . h}, E_{3 . h}\right)$ has $R_{3 . h}$-branching bounded by $2^{n} \cdot m$ for all $h<\omega$.

Proof. It is sufficient to show that each $t \in W_{3 . j-1}$, for $j \leq h$, has at most $2^{n} \cdot m$ immediate $R_{3 . j}$-successors. By construction, we add at most $m$-many immediate $R_{3 . j}$-successors to $t$ for formulas of the form $\square \psi \in \operatorname{Sub}(\varphi)$. Each $y \in E_{3 . j}(t)$ also needs at most $m$-many immediate $R_{3 . j}$-successors to witness $\square$-formulas. Since there are at most $2^{n}$-many such $y$ (including $t$ itself), we must add an immediate $R_{3 . j}$ successor to $t$ for commutativity for at most $2^{n} \cdot m$ points. Thus, $t$ has at most $2^{n} \cdot m$ immediate $R_{3 . j}$-successors.

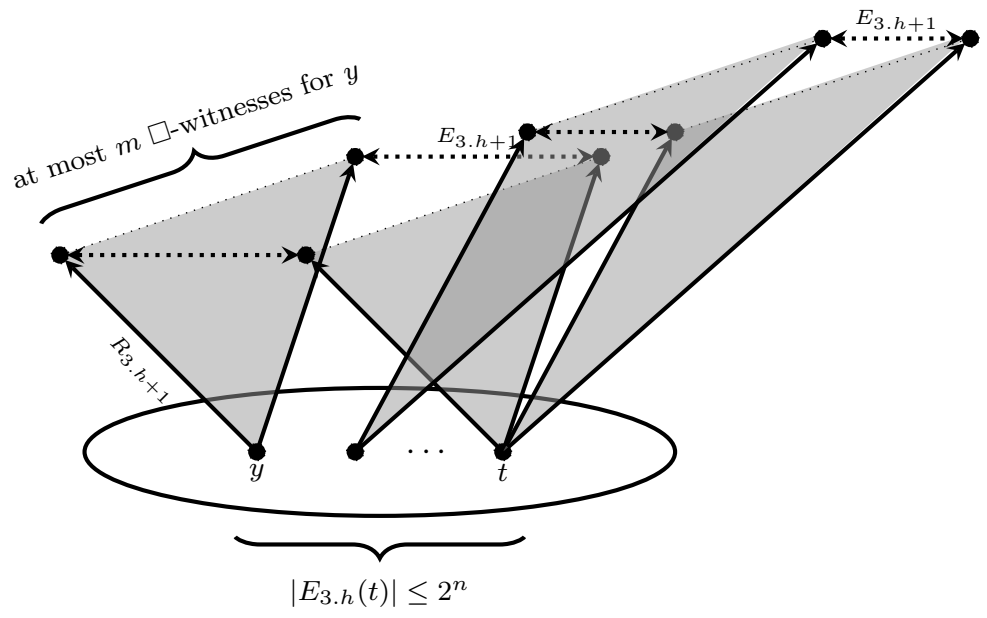

Lemma 6.12. $\mathfrak{F}_{3 . h}=\left(W_{3 . h}, R_{3 . h}, E_{3 . h}\right)$ has $R_{3 . h}$-depth bounded by $2^{n} \cdot m+1$ for all $h<\omega$.

Proof. By construction, to make an immediate vertical move from some cluster $E_{3 . h}(t)$ to another cluster $E_{3 . h}(u)$ (with $t \neq u$ ), there must be some point $x \in E_{3 . h}(t)$ and formula $\square \psi \in \Sigma^{\square}(x)$ requiring a witness $y$, where $y \in E_{3 . h}(u), x R_{3 . h} y$, and $y$ is added in the $\square$-step of the construction. Starting from the bottom cluster $E_{3 . h}\left(t_{0}\right)$, by Lemma 6.9 (7), each of our $m$-many $\square$-formulas can be witnessed at most $2^{n}$ times in clusters along an $R_{3 . h}$-chain. Thus, we add at most $2^{n} \cdot m$ elements to an $R_{3 . h}$ chain originating from this cluster, with the total length of the chain (including the starting point) being at most $2^{n} \cdot m+1$.

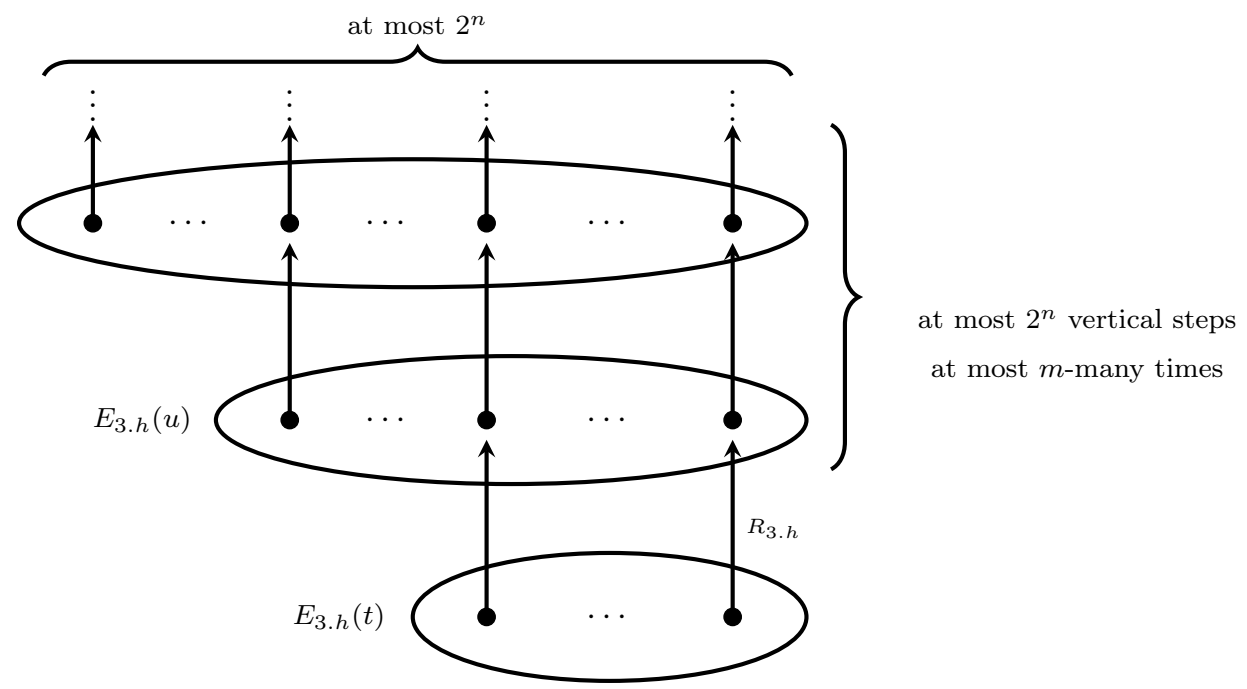

Lemma 6.13. There is $h \in \omega$ such that $\mathfrak{F}_{3 . h^{\prime}}=\mathfrak{F}_{3 . h}$ for all $h^{\prime} \geq h$.

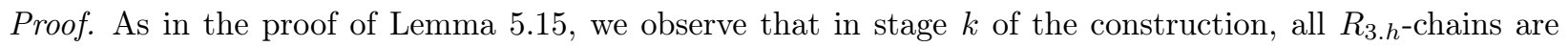
bounded by $k$. Since, by Lemma 6.12 , the $R_{3 . h}$-depth of $\mathfrak{F}_{3 . h}$ is bounded by $2^{n} \cdot m+1$, we have $\mathfrak{F}_{3 \cdot h^{\prime}}=\mathfrak{F}_{2^{n} \cdot m+1}$ for all $h^{\prime} \geq 2^{n} \cdot m+1$. 
Set $\mathfrak{F}_{3}=\left(W_{3}, R_{3}, E_{3}\right)$ where

$$
W_{3}=W_{3 . h}, \quad R_{3}=R_{3 . h}, \quad E_{3}=E_{3 . h},
$$

and $h$ is as in Lemma 6.13. As an immediate consequence of Lemma 6.8, we obtain:

Lemma 6.14. $\mathfrak{F}_{3}=\left(W_{3}, R_{3}, E_{3}\right)$ is a finite $\mathrm{M}^{+}$Grz-frame.

Finally, we verify that our frame validates precisely the formulas we want it to. Define a valuation $\nu_{3}$ on $W_{3}$ by $\nu_{3}(p)=\left\{t \in W_{3}: \hat{t} \in \nu_{2}(p)\right\}$ for $p \in \operatorname{Sub}(\varphi)$ and $\nu_{3}(q)=\varnothing$ for variables $q$ not occurring in $\varphi$.

Lemma 6.15 (Truth Lemma). For $x \in W_{3}$ and $\psi \in \operatorname{Sub}(\varphi)$,

$$
\left(\mathfrak{F}_{2}, \widehat{x}\right) \vDash_{2} \psi \Leftrightarrow\left(\mathfrak{F}_{3}, x\right) \vDash_{3} \psi \text {. }
$$

Proof. The proof is by induction on the complexity of $\psi$ and again we only show the cases where $\psi=\forall \psi_{1}$ or $\psi=\square \psi_{1}$.

Suppose $\psi=\forall \psi_{1}$. If $\widehat{x} \forall_{2} \forall \psi_{1}$, then $\forall \psi_{1} \in \Sigma^{\forall}(x)$, so at some point in the construction of $\mathfrak{F}_{3}$ we add $s$ to $W_{3}$ and $(x, s)$ to $E_{3}$ where $\widehat{s} \forall_{2} \psi_{1}$. By the inductive hypothesis, $s \not_{3} \psi_{1}$, hence $x \forall_{3} \forall \psi_{1}$. Conversely, if $x \forall_{3} \forall \psi_{1}$, then there is $w \in W_{3}$ with $x E_{3} w$ and $w \forall_{3} \psi_{1}$. By the inductive hypothesis, $\widehat{w} \forall_{2} \psi_{1}$, and by Lemma 6.9 (1), $x E_{3} w$ implies $\widehat{x} E_{2} \widehat{w}$, so $\widehat{x} \forall_{2} \forall \psi_{1}$.

Suppose $\psi=\square \psi_{1}$. If $\widehat{x} \not_{2} \square \psi_{1}$, then either $\widehat{x} \not_{2} \psi_{1}$ or $\widehat{x} \vDash_{2} \psi_{1}$. If $\widehat{x} \not_{2} \psi_{1}$, then by the inductive hypothesis we have $x \nvdash_{3} \psi_{1}$, hence $x \nvdash_{3} \square \psi_{1}$. If $\widehat{x} \vDash_{2} \psi_{1}$, then $\square \psi_{1} \in \Sigma^{\square}(x)$, so at some point in the construction of $\mathfrak{F}_{3}$ we add $s$ to $W_{3}$ and $(x, s)$ to $R_{3}$ where $\widehat{s} \not_{2} \psi_{1}$. By the inductive hypothesis, $s \forall_{3} \psi_{1}$, hence $x \forall_{3} \square \psi_{1}$. Conversely, if $x \forall_{3} \square \psi_{1}$, then there is $w \in W_{3}$ with $x R_{3} w$ and $w \forall_{3} \psi_{1}$. By the inductive hypothesis, $\widehat{w} \forall_{2} \psi_{1}$, and by Lemma $6.9(5), x R_{3} w$ implies $\widehat{x} R_{2} \widehat{w}$, so $\widehat{x} \not_{2} \square \psi_{1}$.

The three steps of our construction yield our desired result:

Theorem 6.16. $\mathrm{M}^{+} \mathrm{Grz}$ has the finite model property.

As an immediate corollary to Theorem 6.16, we obtain:

Corollary 6.17. $\mathrm{M}^{+} \mathrm{Grz}$ is decidable.

Remark 6.18. Another consequence of Theorem 6.16 is that $\mathrm{M}^{+} \mathrm{Grz}$ is the monadic fragment of the predicate modal logic obtained by adding to QGrz the Gödel translation of Casari's formula Cas (cf. Remark 5.19).

\section{REFERENCES}

[1] M. A. Abashidze. Some properties of Magari algebras. In Studies in logic and semantics, pages 111-127. Metsniereba, Tbilisi, 1981. In Russian.

[2] G. Bezhanishvili. Varieties of monadic Heyting algebras. I. Studia Logica, 61(3):367-402, 1998.

[3] G. Bezhanishvili. Varieties of monadic Heyting algebras. II. Duality theory. Studia Logica, 62(1):21-48, 1999.

[4] G. Bezhanishvili. Varieties of monadic Heyting algebras. III. Studia Logica, 64(2):215-256, 2000.

[5] G. Bezhanishvili and L. Carai. A temporal interpretation of intuitionistic quantifiers. In TACL 2019. https://math.unice. fr/tacl/2019/contributed-talks.html 2019.

[6] G. Boolos. On systems of modal logic with provability interpretations. Theoria, 46(1):7-18, 1980.

[7] G. Boolos. The logic of provability. Cambridge University Press, Cambridge, 1993.

[8] R. A. Bull. A modal extension of intuitionist logic. Notre Dame J. Formal Logic, 6:142-146, 1965.

[9] R. A. Bull. MIPC as the formalisation of an intuitionist concept of modality. J. Symbolic Logic, 31:609-616, 1966.

[10] A. Chagrov and M. Zakharyaschev. Modal logic, volume 35 of Oxford Logic Guides. The Clarendon Press, Oxford University Press, New York, 1997. Oxford Science Publications.

[11] L. L. Esakia. Topological Kripke models. Dokl. Akad. Nauk SSSR, 214:298-301, 1974.

[12] L. L. Esakia. On modal companions of superintuitionistic logics. VII Soviet Symposium on Logic, Kiev, 1976. (Russian).

[13] L. L. Esakia. On the variety of Grzegorczyk algebras. In Studies in nonclassical logics and set theory (Russian), pages 257-287. "Nauka", Moscow, 1979.

[14] L. L. Esakia. Heyting Algebras I: Duality Theory. Metsniereba, Tbilisi, 1985. (Russian).

[15] L. L. Esakia. Provability logic with quantifier modalities. In Intensional logics and the logical structure of theories (Telavi, 1985), pages 4-9. Metsniereba, Tbilisi, 1988. (Russian).

[16] K. Fine. Logics containing K4. I. J. Symbolic Logic, 39:31-42, 1974.

[17] G. Fischer-Servi. On modal logic with an intuitionistic base. Studia Logica, 36(3):141-149, 1977.

[18] G. Fischer-Servi. The finite model property for MIPQ and some consequences. Notre Dame J. Formal Logic, 19(4):687-692, 1978.

[19] G. Fischer-Servi. Semantics for a class of intuitionistic modal calculi. Polish Acad. Sci. Inst. Philos. Sociol. Bull. Sect. Logic, 7(1):26-30, 1978. 
[20] D. M. Gabbay, A. Kurucz, F. Wolter, and M. Zakharyaschev. Many-dimensional modal logics: Theory and applications, volume 148 of Studies in Logic and the Foundations of Mathematics. North-Holland Publishing Co., Amsterdam, 2003.

[21] R. Goldblatt. Arithmetical necessity, provability and intuitionistic logic. Theoria, 44(1):38-46, 1978.

[22] C. Grefe. Fischer Servi's intuitionistic modal logic has the finite model property. In Advances in modal logic, Vol. 1 (Berlin, 1996), volume 87 of CSLI Lecture Notes, pages 85-98. CSLI Publ., Stanford, CA, 1998.

[23] G. K. Japaridze. Arithmetic completeness of provability logic with quantifier modalities. Soobshch. Akad. Nauk Gruzin. SSR, 132(2):265-268, 1988.

[24] G. K. Japaridze. Decidable and enumerable predicate logics of provability. Studia Logica, 49(1):7-21, 1990.

[25] A. V. Kuznetsov and A. Yu. Muravitsky. Provability as modality. In Actual Problems of Logic and Methodology of Science, pages 193-230. Naukova Dumka, Kiev, 1980. (Russian).

[26] J. C. C. McKinsey and A. Tarski. Some theorems about the sentential calculi of Lewis and Heyting. J. Symbolic Logic, 13:1-15, 1948.

[27] F. Montagna. The predicate modal logic of provability. Notre Dame J. Formal Logic, 25(2):179-189, 1984.

[28] A. Monteiro and O. Varsavsky. Algebras de Heyting monàdicas. pages 50-51, 1957.

[29] H. Ono. On some intuitionistic modal logics. Publ. Res. Inst. Math. Sci., 13(3):687-722, 1977/78.

[30] H. Ono. Some problems in intermediate predicate logics. Rep. Math. Logic, (21):55-67 (1988), 1987.

[31] H. Ono and N.Y. Suzuki. Relations between intuitionistic modal logics and intermediate predicate logics. Rep. Math. Logic, (22):65-87, 1988 .

[32] A. N. Prior. Time and modality, volume 8. Greenwood Press, 1955.

[33] H. Rasiowa and R. Sikorski. The mathematics of metamathematics. Monografie Matematyczne, Tom 41. Państwowe Wydawnictwo Naukowe, Warsaw, 1963.

[34] R. M. Solovay. Provability interpretations of modal logic. Israel J. Math., 25(3-4):287-304, 1976.

New Mexico State University

Email address: guram@nmsu.edu

New Mexico State University

Email address: kleifest@nmsu.edu

University of Amsterdam

Email address: ilin.juli@gmail.com 